\title{
Aerodynamic Analysis of a Generic Fighter with a Chine Fuselage/Delta Wing Configuration Using Delayed Detached-Eddy Simulation
}

\author{
Tiger L. Jeans ${ }^{1}$ \\ Department of Aeronautics, U.S. Air Force Academy, Colorado Springs, CO 80840 \\ David R. McDaniel ${ }^{2}$ \\ Air Force Seek Eagle Office, Eglin Air Force Base, Fl 32542 \\ Russell M. Cummings ${ }^{3}$ \\ Department of Aeronautics, U.S. Air Force Academy, Colorado Springs, CO 80840 \\ and \\ William H. Mason ${ }^{4}$ \\ Virginia Polytechnic Institute and State University, Blacksburg, VA 24061
}

The Modular Transonic Vortex Investigation (MTVI) program at NASA Langley Research Center investigated the transonic characteristics of generic fighter configurations with chined fuselages and delta wings. Previous experiments show that the fuselage and leading edge vortex interactions are detrimental to the vehicle's aerodynamic characteristics for angles of attack greater than $23^{\circ}$ at low angles of sideslip. This is largely due to abrupt asymmetric vortex breakdown, which leads to pronounced pitch-up and significant nonlinearities in lateral stability that could result in roll departure. An improved understanding of the exact origins of this nonlinear behavior would improve future fighter design, and predictive capabilities of such nonlinearities could drastically reduce the cost associated with flight testing new or modified aircraft. The nonlinearities experienced by the MTVI configuration at 30 degrees angle of attack, Reynolds number of $2.68 \times 10^{6}$, and Mach number of 0.4 are computed using Delayed Detached-Eddy Simulation. Computational predictions of rolling moment compare very well with previous wind tunnel experiments at the same conditions, including the abrupt, nonlinear increase in rolling moment as a function of sideslip angle at small sideslip angles. A detailed investigation of the CFD data confirms that this nonlinearity is due to a rapid change in the flow field structures from symmetric to asymmetric vortex breakdown.

\section{Nomenclature}

$\begin{array}{ll}a & =\text { speed of sound } \\ b & =\text { wing reference span, } 19.20 \mathrm{in} \\ C_{D} & =\text { stability-axis drag coefficient, } \frac{\text { Drag }}{q_{\infty} S} \\ C_{L} & =\text { stability-axis lift coefficient, } \frac{\text { Lift }}{q_{\infty} S}\end{array}$

\footnotetext{
${ }^{1}$ Visiting Researcher, Department of Aeronautics, Member AIAA

${ }^{2}$ Aerospace Engineer, Air Force Seek Eagle Office, Member AIAA

${ }^{3}$ Professor, Department of Aeronautics, Associate Fellow AIAA

${ }^{4}$ Professor, Department of Aerospace and Ocean Engineering, Associate Fellow AIAA 


$$
\begin{aligned}
& C_{l} \quad=\text { body-axis rolling-moment coefficient, } \frac{\text { RollingMoment }}{q_{\infty} S b} \\
& C_{m} \quad=\text { body-axis pitching-moment coefficient, } \frac{\text { PitchingMoment }}{q_{\infty} S \bar{c}} \\
& C_{n} \quad=\text { body-axis yawing-moment coefficient, } \frac{\text { YawingMoment }}{q_{\infty} S b} \\
& C_{p} \quad=\text { static pressure coefficient, } \frac{p-p_{\infty}}{q_{\infty}} \\
& C_{p t} \quad=\text { total pressure coefficient, }=\frac{p_{t}-p_{t \infty}}{q_{\infty}} \\
& C_{Y} \quad=\text { body-axis side force coefficient, } \frac{\text { SideForce }}{q_{\infty} S} \\
& \bar{c} \quad=\text { wing mean aerodynamic chord, } 12.97 \text { in } \\
& l=\text { body length, } 32.48 \text { in } \\
& M=\text { Mach number, } U / a \\
& p \quad=\text { pressure } \\
& p_{t} \quad=\text { total pressure } \\
& q \quad=\text { dynamic pressure, } \frac{\rho U^{2}}{2} \\
& \operatorname{Re}_{\bar{c}}=\text { Reynolds number based on the mean aerodynamic chord, } \frac{\rho U_{\infty} \bar{c}}{\mu} \\
& S \quad=\quad \text { reference wing area, } 208.19 \mathrm{in}^{2} \\
& U \quad=\text { velocity magnitude } \\
& \alpha \quad=\text { angle of attack, deg } \\
& \beta \quad=\text { angle of sideslip, deg } \\
& \rho \quad=\quad \text { fluid density } \\
& \mu \quad=\text { dynamic viscosity }
\end{aligned}
$$

Subscript:

$\infty \quad=$ free-steam conditions

\section{Introduction}

$\mathrm{I}_{\mathrm{d}}^{\mathrm{n}}$ $\mathrm{n}$ recent years many advanced fighters, such as the F-22 and F-35, have incorporated a chine-shaped forebody designed to minimize observables. The chine generally results in stronger forebody vortices being shed compared to traditional fighters with smooth forebodies. For certain flight conditions these vortices interact with the wing leading edge vortices, and improve the maneuvering lift capabilities. ${ }^{1}$ However, for other flight conditions these vortex interactions are detrimental to the aerodynamic characteristics of the vehicle. This is especially true when the fighter experiences moderate to low angles of sideslip at angles of attack in excess of $25^{\circ}$. Under these conditions abrupt asymmetric vortex breakdown leads to pronounced pitch-up and significant nonlinearities in lateral stability that could result in roll departure. ${ }^{2}$

The vortex interactions of chine fuselage/delta wing configurations have been extensively investigated experimentally. ${ }^{1-8}$ Erickson and Brandon ${ }^{1}$ were amongst the first to investigate the nonlinear aerodynamic and stability characteristics of a chine-shaped forebody and delta wing configuration. They concluded that at high angles of attack the chine-wing vortex system was susceptible to severe asymmetries in the core breakdown positions in sideslip. LeMay and Rogers ${ }^{3}$ studied pneumatic vortex flow control on a similar configuration and give two possible reasons for the asymmetric vortex breakdown. First, the chine-wing vortex system interacts such that the chine vortex is pulled down and under the wing vortex, and therefore, experiences a severe adverse pressure gradient on the wing surface at higher angles of attack. Second, sideslip reduces the effective sweep angle on the windward wing which increases the strength of the windward vortex system thereby promoting vortex breakdown, while the opposite is true for the leeward wing. Grismer and Nelson ${ }^{4}$ studied the aerodynamic effects of a double 
delta wing configuration in sideslip, and they too attributed the asymmetric vortex breakdown to the decrease and increase of the effective sweep angle on the windward and leeward sides, respectively. Rao and Bhat ${ }^{2,5,6}$ investigated methods to decouple the forebody and leading edge vortices. For the purposes of their study they developed the Modular Transonic Vortex Interaction (MTVI) body shape. For their experiments Mach number varied from 0.4 to 1.2 and Reynolds number ranged from $0.6 \times 10^{6}$ to $2.24 \times 10^{6}$ based on the mean aerodynamic chord. Rao and Bhat found that in general leading edge flaps were very effective at decoupling the forebody and leading edge vortices, removing the nonlinearities in lateral stability at high alpha. They also found that twin vertical tails were effective at accomplishing the same result, while a single vertical tail was somewhat effective. Hall $^{7}$ studied the impact of the fuselage cross section on the stability of the MTVI body shape without employing leading edge flaps or vertical tails. He tested a $30^{\circ}$ and $100^{\circ}$ chine cross-section in the upright and inverted positions. These experiments were conducted at Mach $=0.4$ for Reynolds numbers ranging from $2.61 \times 10^{6}$ to $2.84 \times 10^{6}$. Hall found significant nonlinearities in the rolling moment for all cross sections for $\alpha \geq 23^{\circ}$ and $-5^{\circ}<\beta<$ $5^{\circ}$. He concluded that in general the roll stability was much better for the sharper chine angle cross section and the directional stability was significantly better for the upright configurations.

Significant computational efforts have also been made to fully understand the flow over fighters with chine forebodies. ${ }^{9-20}$ Ravi and Mason ${ }^{9,10}$ studied the effects of the isolated fuselage on directional stability at high alpha and sideslip using both the Euler equations and Reynolds equations employing the Baldwin-Lomax turbulence model. They also developed an analytic model for systematically studying forebody aerodynamics for a family of forebody cross-sections. In addition, Agosta-Greenman et al. ${ }^{11}$ computationally investigated the effects of tangential slot blowing on an isolated chined forebody using thin-layer RANS equations. Throughout the mid 1990's there was a significant joint effort by NASA LaRC, Lockheed Martin and Boeing to access the ability of using Euler-based Computational Fluid Dynamic (CFD) codes to predict the aerodynamic characteristics at moderate to high alpha for preliminary design. The MTVI configuration was analyzed using the Cartesian-grid SPLITFLOW code, ${ }^{15}$ multiblock structured grid TEAM code,${ }^{16}$ multi-block overset structured-grid OVERFLOW code,${ }^{17}$ and unstructured-grid USM3D code. ${ }^{18}$ The general conclusion from this effort was that Euler solutions can provide meaningful guidelines for preliminary design of flight vehicles when dominated by vortical flow. However, for all of these studies the MTVI configurations included leading edge flaps, vertical tails, or some combination thereof, and therefore did not experience the significant aerodynamic nonlinearities.

Although there has been a significant computational effort to model the flow around the MTVI configuration, to the authors knowledge no one has attempted to compute the nonlinear lateral-directional behavior measured by Hall. The goal of this paper is to utilize Delayed Detached-Eddy Simulation ${ }^{21}$ (DDES) to predict these nonlinear aerodynamic characteristics, and then to use the detailed CFD results to offer new insight on the root cause of these aerodynamic instabilities. As such, an improved understanding of the exact origins of this nonlinear behavior would improve future fighter design, and predictive capabilities of such nonlinearities could drastically reduce the cost associated with flight testing new or modified aircraft. In addition, this work is in support of a larger aircraft stability and control project ongoing at the United State Air Force Academy Modeling and Simulation Research Center (USAFA M\&SRC) and the United States Air Force Seek Eagle Office (USAF SEO). The goal of this project is to develop lower order predictive models for aerodynamic loads of complete aircraft configurations. The results of this study will be used to help develop and validate these models. The MTVI geometry is an excellent test case due to the nonlinear aerodynamics that occurs well within the flight envelope.

The MTVI configuration employed for this study utilized the $30^{\circ}$ chine fuselage without leading edge flaps or vertical tails. A schematic of the overall shape along with the fuselage cross-section are shown in Fig. 1. The MTVI body has a span of 29.90 inches and a length of 32.48 inches. The wing is comprised of a cropped-delta planform with a $60^{\circ}$ leading edge sweep and a NACA 65-005 airfoil section modified with double-arc section forward of the maximum thickness and sharp leading edges. ${ }^{5}$ Non-dimensional forces and moments are calculated relative to the standard body axis system with the origin located at the quarter chord of the mean aerodynamic chord, located 20.36 inches from the nose.

\section{Computational Methodology}

\section{A. Flow Solver}

Computations were performed using the commercially available flow solver Cobalt ${ }^{22}$ which solves the unsteady, three-dimensional, compressible Reynolds Average Navier-Stokes (RANS) equations. Cobalt is a cell-centered, finite volume based code applicable to arbitrary cell topologies including prisms, tetrahedra and hexahedrals. 
Second order accuracy in space is achieved using the exact Riemann solver of Gottleib and Groth, ${ }^{23}$ and least squares gradient calculations using QR factorization. To advance the discretized system a point-implicit method using analytic first-order inviscid and viscous Jacobians is used. A Newton sub-iteration method is used in the solution of the system of equations to improve time accuracy of the point-implicit method. The method is second order accurate in time. Tomaro et al. ${ }^{24}$ converted the code from explicit to implicit, enabling CFL numbers as high as $10^{6}$.

Time-dependent computations were performed using the Delayed Detached-Eddy Simulation technique proposed by Spalart et al.. ${ }^{21}$ Cobalt has been previously used in conjunction with DES to successfully model similar high angle of attack flows. ${ }^{25-27}$ DDES is conceptually similar to the original DES technique proposed by Spalart et al., ${ }^{28}$ where the entire boundary layer is treated using a RANS model and highly separated regions are treated using Large Eddy Simulation (LES). This results in a numerically feasible approach that combines the most favorable elements of each method. Both the original DES model and the DDES model are based on the Spalart-Allmaras ${ }^{29}$ (SA) one-equation RANS turbulence model. When the wall destruction term in the SA model is balanced with the production term $\hat{S}$, the eddy viscosity becomes proportional to $\hat{S} d^{2}$, where $d$ is the distance to the nearest wall. In the Smagorinski LES model the subgrid-scale turbulent viscosity is described by $v_{S G S} \propto \hat{S} \Delta^{2}$, where $\Delta=\max (\Delta x, \Delta y, \Delta z)$. Therefore, if $d$ in the wall destruction term of the SA model is replaced with $\Delta$ it will act as the Smagorinski LES model. To exhibit both RANS and LES behavior, $d$ in the SA model is replaced by the DES length scale $\tilde{d}$. The primary difference between DDES and DES is in the definition of the limiter $\widetilde{d}$, where the DDES formulation is intended to remove the ambiguous-grid issue emphasized by Menter and Kuntz. ${ }^{30}$ Details of the reformulation of $\tilde{d}$ can be found in Spalart et al., ${ }^{21}$ however, it is important to note that this new formulation does not represent a minor adjustment within DES. In the original DES formulation $\tilde{d}$ depended only on the grid, but in the current DDES formulation $\widetilde{d}$ depends on the eddy-viscosity field. It should also be noted that for the current study the Spalart-Allmaras Rotational Correction ${ }^{31}$ (SARC) turbulence model was implemented. The SARC turbulence model includes modifications to the original SA model to account for the effect of system rotation and/or streamline curvature.

\section{B. Mesh Generation}

Generating a quality mesh is essential for an accurate representation of the flow conditions. The meshes used for the current study were created using Solidmesh $3 \mathrm{D}^{32}$, which is currently being developed at the Mississippi State University. Solidmesh 3D is an Advancing Front Local Reconnection (AFLR) unstructured grid generator. An unstructured triangular mesh was generated over the surfaces of the domain and volume elements are then inflated.

A total of four meshes were generated for this study. For each of the meshes, the first node height was chosen using the approach of Cummings et al. ${ }^{33}$ such that the average $\mathrm{y}^{+}$was below 0.5 . This ensures an adequate volume mesh that correctly models the boundary layer. The primary mesh, which will be referred to at the medium density mesh (MDM), consisted of 10.9 million cells. The medium density surface mesh is shown in Fig. 1 (a), and a crosssectional and side view of the mesh elements at $x / l=0.88$ and $y / l=0.06$, respectively, are shown in Fig. 2 (a) and (b). As shown in Fig. 2 (a), planar mesh controls were implemented above the body such that elements were clustered just above the fuselage and wing to ensure that the forebody and leading edge vortices were properly convected downstream. To conduct a time step and grid sensitivity study, two additional meshes were generated with the same topology as the MDM, but the cell spacing was altered such that the total cell counts were approximately 5.5 million cells (referred to as the low density mesh, LDM) and 22.6 million cells (referred to as the high density mesh, HDM). A limited number of simulations were completed using a final mesh (referred to as the very fine mesh, VFM) that consisted of 23.3 million cells. Cross-sectional and side views of the mesh elements are shown in Fig. 2 (c) and (d). The primary difference between this mesh and the others was that the number of cells through the chine and wing vortex cores was significantly increased.

\section{Computation Setup}

The computational domain was rectangular in shape with the MTVI geometry centrally located. The minimal distance from the body to each of the outer boundaries was $10 \mathrm{l}$. The no-slip adiabatic wall boundary condition was employed for the body surface and the modified Riemann-invariant condition was implemented as the farfield

American Institute of Aeronautics and Astronautics

DISTRIBUTION A. Approved for public release, distribution unlimited. 
boundary. It should also be noted that the sting matching the one used in the previous wind tunnel experiments ${ }^{5-7}$ was incorporated into the geometry. The no-slip adiabatic wall condition without force accounting was implemented on the sting.

Two distinct initial conditions were used for the simulations. The first, referred to as initial condition A, was to initialize the entire domain with the free steam conditions. The second, referred to as initial condition B, was to initialize the entire domain using the flow field predicted by a previous simulation at similar conditions. For example, the flow field at $\alpha=25^{\circ}$ and $\beta=0^{\circ}$ was used to initialize the simulation at $\alpha=30^{\circ}$ and $\beta=0^{\circ}$. It will be shown in the Results and Discussion section that for certain flight conditions initial condition A and B result in different solutions.

Time dependent computations were performed at $\operatorname{Re}_{\bar{c}}=2.68 \times 10^{6}$ and $M_{\infty}=0.40$, which correspond to the experiments of $\mathrm{Hall}^{7}$ for the $30^{\circ}$ chine forebody. Simulations were carried out for nominally 7,000 to 11,000 time steps depending on the flow field conditions, and the first 2,000 to 6,000 time steps were eliminated to remove transients. This ensured a minimum of 5000 time steps of useable solution. In addition, three Newton sub-iterations were used to ensure that the flow solution was converged at every time step.

\section{Grid Refinement and Time Step Study}

When predicting time dependant flow fields it important to ensure that the mesh density is sufficient such that the solution is spatially converged and the time step is such that the temporal aspects of the flow field have been sufficiently resolved. Cummings et al. ${ }^{33}$ showed that for time-dependent flows a joint time step/grid density study is required because different meshes obtain time independence at different time step values. They developed a method for ensuring that both temporal and spatial independence have been achieved, which is based on analyzing the frequency content of an appropriate flow field variable (e.g. integrated forces or moments or pressure at a specific location) using Power Spectrum Density (PSD) analysis. For a given mesh, the time step should be sufficiently small such that dominant flow field frequencies are time step independent.

A grid/time step study was performed for the geometry at $\alpha=30^{\circ}$ and $\beta=0^{\circ}$. Since the study was performed at zero sideslip, symmetry was assumed and only half the domain was modeled. Simulations were completed using the low, medium and high density meshes for dimensionless time steps ranging from $t^{*}=0.08$ to $t^{*}=0.005$, where $t^{*}$ is defined by,

$$
t^{*}=\frac{\Delta t U_{\infty}}{\bar{c}}
$$

For $t^{*}=0.01,100$ time steps are required for the free steam to travel over the mean aerodynamic chord of the wing. To determine the flow field frequency content, a PSD analysis of pitching moment was completed for each solution using MATLAB. PSD results for the MDM at various time steps are given in Fig. 3 (a), which show a dominate flow field frequency near $S t=7$, where the Strouhal number is defined as,

$$
S t=\frac{f \bar{c}}{U_{\infty}}
$$

Plotted in Fig. 3 (b) are primary frequencies (for $S t>2$ ) for all of the CFD simulations, which show that $t^{*}=0.01$ is sufficient for the MDM. In addition, a comparison of time averaged lift, drag and pitching moment with mesh size is shown in Fig. 3 (c) for $t^{*}=0.01$, which show that integrated forces and moments have converged using the MDM. These results allow one to conclude with a high level of confidence that the solutions are spatially and temporally converged using the MDM in conjunction with $t^{*}=0.01$.

\section{Results and Discussion}

All of the CFD simulations were performed at the Arctic Region Supercomputing Center (ARSC) on Midnight, a Sun cluster comprised of 2312 Opteron processors with a 68 TB Lustre file system. The pertinent CFD simulations included in the final dataset are listed in Table 1. All of these simulations were performed at $\operatorname{Re}_{\bar{c}}=2.68 \times 10^{6}$ and $M_{\infty}=0.40$ using $t^{*}=0.01$. In addition to the aircraft orientation relative to the free stream, also listed in Table 1 are the corresponding initial condition and mesh for each simulation. Simulations with the same initial condition and

American Institute of Aeronautics and Astronautics

DISTRIBUTION A. Approved for public release, distribution unlimited. 
mesh are grouped together in the Case column, which will be used for identification purposes throughout the remainder of the article.

Table 1. Pertinent computational fluid dynamics cases included in final dataset.

\begin{tabular}{|c|c|c|c|c|c|}
\hline Simulation & $\alpha(\mathrm{deg})$ & $\beta(\mathrm{deg})$ & $\begin{array}{c}\text { Initial } \\
\text { Condition }\end{array}$ & Mesh & Case \\
\hline 1 & 15 & 0.00 & A & MDM & 1 \\
\hline 2 & 20 & 0.00 & A & MDM & 1 \\
\hline 3 & 25 & 0.00 & A & MDM & 1 \\
\hline 4 & 29 & 0.00 & A & MDM & 1 \\
\hline 5 & 30 & 0.00 & A & MDM & 1 \\
\hline 6 & 30 & 0.25 & A & MDM & 1 \\
\hline 7 & 30 & 0.50 & A & MDM & 1 \\
\hline 8 & 30 & 0.75 & A & MDM & 1 \\
\hline 9 & 30 & 0.85 & A & MDM & 1 \\
\hline 10 & 30 & 1.00 & A & MDM & 1 \\
\hline 11 & 30 & 1.15 & A & MDM & 1 \\
\hline 12 & 30 & 1.25 & A & MDM & 1 \\
\hline 13 & 30 & 1.50 & A & MDM & 1 \\
\hline 14 & 30 & 2.00 & A & MDM & 1 \\
\hline 15 & 30 & 2.50 & A & MDM & 1 \\
\hline 16 & 30 & 3.00 & A & MDM & 1 \\
\hline 17 & 30 & 3.50 & A & MDM & 1 \\
\hline 18 & 30 & 5.00 & A & MDM & 1 \\
\hline 19 & 30 & 0.00 & B & MDM & 2 \\
\hline 20 & 30 & 0.25 & B & MDM & 2 \\
\hline 21 & 30 & 0.85 & B & MDM & 2 \\
\hline 22 & 30 & 1.00 & B & MDM & 2 \\
\hline 23 & 30 & 2.00 & B & MDM & 2 \\
\hline 24 & 25 & 0.00 & A & VFM & 3 \\
\hline 25 & 30 & 0.00 & A & VFM & 3 \\
\hline 26 & 30 & 0.85 & A & VFM & 3 \\
\hline & & & & & \\
\hline
\end{tabular}

\section{A. Zero Sideslip}

This section describes in detail the results of all the simulations completed at zero sideslip.

\section{Overall Force and Moment Predictions}

The primary means of validating the DDES predictions is by comparison of overall force and moment coefficients with the experimental data in Ref. 7. A typical CFD time history of total force and moment coefficients are shown in Fig. 4. Once transients are properly removed, the data was averaged using output at every time step to determine mean total force and moments coefficients that could be compared to experimental results.

Time averaged lift, drag and pitching moment coefficients are plotted in Fig. 5 verses angle of incidence for zero sideslip. All of the DDES predictions are in excellent agreement with experiment for $\alpha \leq 25^{\circ}$, however, at incidence angles greater than this the agreement differs based on the initial conditions used for the DDES simulation. At $\alpha=$ $30^{\circ}$, initializing the CFD domain to free stream conditions (Case 1 and Case 3) resulted in solutions that under predicts the experimental lift and drag by approximately $12 \%$ and $13 \%$, respectively. Whereas, initializing the CFD domain using the DDES solution at $\alpha=25^{\circ}$ (Case 2) resulted in lift and drag predictions that were again in excellent agreement with experiment. The cause of this discrepancy is discussed further in the next section. 


\section{Critical Flow Features}

This section describes some of the critical flow features seen in the $\alpha=30^{\circ}, \beta=0^{\circ}$ results, such that the discrepancies in the lift and drag predictions for Cases 1,2 and 3 are investigated further. All of the data presented in this section (i.e. Fig. 6 to 8 ) is time averaged using results that were output at every 50 time steps.

Major vortical structures are highlighted in Fig. 6 using the Q vortex identification criterion. ${ }^{34}$ The Q criterion results in a better representation of the main vortical structures than vorticity because it eliminates the influence of the mean shear in the boundary layer and early shear layers near separation. Plotted in Fig. 6 are isosurfaces of Q = $1.0 \times 10^{7} \mathrm{~s}^{-2}$ for all three simulations completed at zero sideslip and $30^{\circ}$ incidence. The isosurfaces are colored by axial velocity with red corresponding to $u=0$ and blue corresponding to $u=-2 u_{\infty}$. It should also be noted that the left side of each figure is the windward side of the body. The primary fuselage and leading edge vortices are clearly evident in all of the figures. As shown in Fig. 6 (a) and (b), the main difference between the Case 1 and 2 results, which both employed the MDM but with different initial conditions, is vortex breakdown. Case 1 predicts symmetric breakdown of both the windward and leeward vortex systems, whereas, Case 2 predicts no vortex breakdown.

A detailed investigation was conducted to determine the effect of the different vortex structures predicted by the Case 1 and 2 on the total body force predictions. Contours of surface pressure $\left(C_{p}\right)$ for both simulations are plotted in Fig. 7 (a) and (b). The vortex breakdown predicted in Case 1 destroys the low pressure region on the upper surface of the wing compared to Case 2. The extended low pressure region predicted by Case 2 could account for the increase lift and drag predictions presented earlier. This is further reinforced with a comparison of axial normal force distributions, $C_{Z}$, which are shown in Fig. 8. The vortex breakdown in the Case 1 solution causes a significant decrease in the normal force distribution from $x / l=0.6$ to $x / l=0.90$.

The differences in the Case 1 and 2 predictions were initially a concern and warranted further investigation. To ensure that the vortex breakdown in Case 1 was not a result of artificial vorticity dissipation due to insufficient mesh density, a third simulation that employed the very fine mesh with initial condition A (Case 3) was completed. However, as shown in Fig. 6, 7, and 8 (c), it also predicted symmetric vortex breakdown, resulting in similar surface pressure and normal force distributions, and hence overall force and moment predictions, as Case 1. Grismer and Nelson ${ }^{35}$ experimentally measured the aerodynamic effects of a pitching double-delta-wing in sideslip, and found a significant hysteresis loop in the lift curve with angle of attack. Their configuration, which exhibits a similar vortex system as the MTVI, resulted in significantly increase lift measurements when pitching upward in incidence compared to pitching downward in incidence for $\alpha>25^{\circ}$. Based on these results it was concluded that the different solutions predicted by Case 1 and 2 was entirely the result of the different initial conditions employed, however, both are physically correct. A comparison of experimental laser sheet data collect by Hall ${ }^{7}$ shows no vortex breakdown at these conditions, which more closely matches Case 2 results. However, these results are likely dependent on the manner in which the experimental data was collected.

\section{B. Sideslip at $3^{\circ}$ Angle of Attack}

This section describes in detail the results of all the simulations completed at $30^{\circ}$ angle of attack with sideslip.

\section{Overall Moment Predictions}

A comparison of DDES predicted and experimentally measured rolling and yawing moment coefficients as a function of $\beta$ are given in Fig. 9. As in Section A1, transients was properly removed and the remaining data was averaged using output at every time step. To highlight the hysteresis in the experimental data both positive and negative sideslip are plotted on the same axis. Computational results are shown for both Case 1 and 2 simulations. It is interesting to note that employing initial conditions A and B has less of an effect on the rolling and yawing moment predictions than lift, drag and pitching moment. As seen in Fig. 9 (a), the DDES rolling moment predictions for both Case 1 and 2 simulations compare very well with experiment, accurately predicting both the location and magnitude of the nonlinearity at approximately $\beta=1.0^{\circ}$. The DDES yawing moment predictions in Fig. 9 (b) are qualitatively similar to the experimental results. DDES correctly predicts both the nonlinearity at $\beta=$ $1.0^{\circ}$ and the magnitude of the upward shift in $C_{n}$ due to this nonlinearity. However, there is a general downward shift in all of the DDES yawing moment predictions between $0.0^{\circ}<\beta<2.0^{\circ}$. The exact cause of this discrepancy is still under investigation. 


\section{Critical Flow Features}

This section presents the critical flow features in all the simulations with sideslip, such that the causes of the rolling moment non-linearity are highlighted. As in Section A2, all of the data presented in this section (Fig. 10 to 15 ) is timed averaged using results that were output at every 50 time steps.

The major vortical structures are again highlighted using the $\mathrm{Q}$ vortex identification criterion. Plotted in Fig. 10 and 11 are isosurfaces of $\mathrm{Q}$ colored by axial velocity at different sideslip angles for Case 1 and 2 simulations, respectively. In general, the topology of the vortical structures for $\beta<1.0^{\circ}$ are similar, and there is a distinct change in the vortical structures for $\beta>1.0^{\circ}$. For all the Case 1simulations at $\beta<1.0^{\circ}$, the fuselage and leading edge vortical structures over both the windward and leeward wings start to intertwine before bursting near the trailing edge. For all the Case 2 simulations at $\beta<1.0^{\circ}$, the fuselage and leading edge vortical structures over both the windward and leeward wings intertwine. Again, the primary difference between the Case 1 and 2 results is the vortex breakdown seen in Case 1. For both Case 1 and 2 simulations there is a distinct asymmetry in the vortical structures between the windward and leeward sides for $\beta>1.0^{\circ}$. Notice from Fig. 10 (c) and Fig. 11 (d), which are both at $\beta=2.0^{\circ}$, that Case 1 and Case 2 predict nearly identical flow fields. The vortical structures over the windward wing burst, while the vortical structures over the leeward wing interact and remain as coherent structures for the full body length. It is these asymmetrical vortex structures that induce the nonlinearities in both rolling and yawing moment.

To investigate the influence of the vortical structures on the surface pressure, contours of surface pressure, $C_{p}$, are plotted in Fig. 12 and 13 for Case 1 and 2 respectively, at the corresponding sideslip angles in Fig. 10 and 11. For all simulations at $\beta<1.0^{\circ}$ there is a low pressure region evident on both the windward and leeward wings due to the leading edge and chine vortices. By comparison of Fig. 12 (a) to Fig. 13 (a) to (c), one can see that for the Case 2 results these low pressure regions are extended much further downstream. This is partially due to the increased interactions of the leading and chine vortex, which pull the chine vortex downward and outboard, thereby causing a greater influence on the surface pressure. In addition, from Fig. 7, 12 and 13 one can see that at $\beta=0.00^{\circ}$ these low pressure regions are symmetric, but as $\beta$ increases to $\beta=0.85^{\circ}$ the leeward low pressure region is diminished, which would account for the negative rolling moment at this sideslip. For $\beta>1.00^{\circ}$ the forward movement of the windward vortex breakdown significantly reduces the size of the windward low pressure region in both the Case 1 and 2 results. In addition, on the leeward side the lack of leeward vortex breakdown increases the size of the low pressure region for the Case 1 results, while the Case 2 results remains relatively unchanged. Conceptually, this rapid change to an asymmetric surface pressure distribution could cause the abrupt increase in rolling moment shown in Fig. 9. As $\beta$ continues to increase beyond $\beta=1.0^{\circ}$, the leeward low pressure region continually diminishes. Closer investigation of Fig. 10 (d) indicates that this is because the leeward vortical structures are displaced further from the body as $\beta$ is increased, and therefore, have less effect on the surface pressure distribution. This would correspond to a decreasing rolling moment with increasing $\beta$ in this region, which is in agreement with Fig. 9.

To further investigate the source of the nonlinear rolling moment behavior, plotted in Fig. 14 are axial distributions of sectional rolling moment, $C_{l}$, where the integrated distribution is equal to the total rolling moment coefficients. Plotted in Fig. 14 (a) are distributions based on Case 1 results and in Fig. 14 (b) are distributions based on Case 2 results. All of the distributions exhibit nearly a zero contribution to the rolling moment along the chined forebody. One exception to this is the Case 1 simulation at $\beta=5.0^{\circ}$, which experiences a small negative rolling moment along much of the nose. The primary differences in the distributions occur along the wing between $x / l=0.5$ and $x / l=0.92$. For $\beta<1.0^{\circ}$ there is generally a negative contribution to the rolling moment along the wing, although, some distributions do experience a small positive contribution between $x / l=0.70$ and $x / l=0.75$. For $\beta>$ $1.0^{\circ}$ there is generally a large positive contribution to the rolling moment along the wing. The distributions increase rapidly and nonlinearly up to a maximum value at approximately $x / l=0.75$, at which point they decline over the remainder of the wing. As sideslip is increased beyond $\beta=1.0^{\circ}$, the maximum value of $C_{l}$ ' decreases from 0.0045 at $\beta=1.0^{\circ}$ to 0.00125 at $\beta=5.0^{\circ}$. It is also interesting to note that the distributions are nearly symmetric about $x / l=$ 0.75 .

The sectional rolling moment distributions are complemented with axial distributions of pressure in Fig. 15 and 16. Distributions are plotted for $x / l=0.55,0.65,0.75$ and 0.85 for each distribution shown in Fig 14 . At $x / l=0.55$ all of the pressure distributions are asymmetric such that a local negative rolling moment is induced. This is due to increased suctions on the windward side by both the wing leading edge and chine vortices. At $x / l=0.65,0.75$, and 0.85 there are significant differences in the distributions between all the simulations where $\beta<1.0^{\circ}$ and $\beta>1.0^{\circ}$. For the simulations with $\beta<1.0^{\circ}$, the distributions are similar to those seen at $x / l=0.55$, whereby the suction peaks induced by the windward vortices induce a small negative local rolling moment. Note that for the Case 2

American Institute of Aeronautics and Astronautics 092407 
simulations $\left(\beta=0.25^{\circ}, 0.85^{\circ}\right.$, and $\left.1.0^{\circ}\right)$, the primary suction peaks are significantly larger over the entire wing compared to the Case 1 simulation $\left(\beta=0.85^{\circ}\right)$, however, the loss of primary suction in the Case 1 simulation occurs on both the windward and leeward sides such that the local rolling moment predictions remain relatively unchanged. For the simulations with $\beta>1.0^{\circ}$, there is a significant loss of primary windward suction at $x / l=0.65,0.75$, and 0.85 due to the breakdown of the windward vortex system. This results in an asymmetric vortex distribution that induces a strong local positive rolling moment. Also notice that this asymmetry is decreased as sideslip increases to $\beta=5.0^{\circ}$ primarily because the primary suction on the leeward side continually diminishes with increasing sideslip.

\section{Comparison of Vortex Properties Prior and Post Asymmetric Breakdown}

One major advantage of CFD over wind-tunnel experimentation is that once you have a verified and validated solution you now have access to the entire flow field that you can analyze. For the current study, one can gain an increased level of understanding as to the cause of the sudden asymmetric vortex breakdown at $\beta=1.0^{\circ}$ by comparing vortex properties pre and post breakdown. In this section a comparison of vortex properties between Case 2 simulations at $\beta=1.0^{\circ}$ and $2.0^{\circ}$ is completed, including the trajectory, total pressure, and static pressure of the vortex cores.

Plotted in Fig. 17 is a comparison of vortex trajectories between $\beta=1.0^{\circ}$ and $2.0^{\circ}$, where the red squares identify the $\beta=1.0^{\circ}$ trajectories and the blue diamonds identify the $\beta=2.0^{\circ}$ trajectories. These trajectories we determined by locating the maximum total pressure depreciation for each vortex. One can notice from the figure that the trajectories of the windward vortex systems are nearly identical in the y-axis direction. The chine vortex is slightly inboard at $\beta=2.0^{\circ}$ from $x / l=0.55$ to breakdown, but it is unlikely that this small difference is the cause of the vortex breakdown. In the z-axis direction, notice that at $\beta=2.0^{\circ}$ the windward chine vortex is pull upward and the leading chine vortex is pulled downward from $x / l=0.55$ to breakdown, however, this difference is still relatively small and it is unclear if this increased vortex interaction is sufficient to induce breakdown. For the leeward vortex system notice that at $\beta=2.0^{\circ}$ the vortices are more outboard and upward from the wing surface, which would account for the above mentioned decrease in suction on the leeward wing with increasing sideslip.

Finally, a comparison of the total pressure, $C_{p t}$, and static pressure, $C_{p}$, through the vortex cores is given in Fig. 18. Again, the red squares represent $\beta=1.0^{\circ}$ results and blue diamonds represent $\beta=2.0^{\circ}$ results. In addition, unfilled symbols represent the chine vortex and filled symbols represent the leading edge vortex. We will first concentrate on the total pressure distributions, which can be used as indicator of vortex strength, where increases in total pressure depreciation indicate a stronger vortex. Both the leeward chine and leading edge vortices decrease in strength with increasing sideslip. On the windward side, the vortices at $\beta=1.0^{\circ}$ and $2.0^{\circ}$ are nearly identical in strength up to $x / l=0.55$. However, for $\beta=2.0^{\circ}$, there is a significant increase in both the chine and leading edge vortex strength from $x / l=0.55$ to breakdown. These findings are in agreement with the conclusions of LeMay and Rogers ${ }^{3}$ and Grismer and Nelson ${ }^{4}$. Concentrating now on the static pressure distributions, both the windward chine and leading edge vortices experience a significant adverse pressure gradient prior to breakdown that is not experienced by any of the other vortices. It is expected that this adverse pressure gradient combined with the increased vortex strength induces breakdown, however the exact cause of this behavior is still under investigation.

\section{Conclusions}

Time accurate Delayed Detached-Eddy Simulations were performed on the MTVI configuration at a Reynolds number of 2.68 million and Mach number of 0.4, for angles of attack up to $30^{\circ}$ and sideslip up to $5.0^{\circ}$. At zero sideslip, lift and drag predictions are in excellent agreement with experiment for the full range of angle of attack investigated. At $30^{\circ}$ angle of attack, rolling moment predictions are in excellent agreement with experiment for sideslip up to $5^{\circ}$, accurately predicting the severely nonlinear aerodynamic behavior experienced by the MTVI geometry at approximately $1^{\circ}$ sideslip. Such predictive capabilities will improve the design of future fighters and could drastically reduce the cost associated with flight testing new or modified aircraft.

Critical flow features were investigated at a level of detail that is difficult to obtain from experiment. The investigation confirmed that the nonlinear aerodynamic behavior is primarily due to abrupt asymmetric vortex breakdown over the windward wing. This resulted in severely asymmetric surface pressure distributions that induce significant positive sectional rolling moments along the wing.

A comparison of vortex properties at $1^{\circ}$ and $2^{\circ}$ sideslip showed only minor changes in vortex trajectory, indicating that this is not the likely cause of the abrupt vortex breakdown. However, at $2^{\circ}$ sideslip, the windward vortex system experienced a significant increase in total pressure depreciation and an adverse pressure gradient that were not experienced by the leeward vortex system or by the windward vortex system at $1^{\circ}$ sideslip. This increase in vortex

American Institute of Aeronautics and Astronautics

DISTRIBUTION A. Approved for public release, distribution unlimited. 
strength combined with the adverse pressure gradient would tend to induce vortex breakdown, but their root cause is still being investigated.

The results of this study will be used to develop and validate lower order stability and control models being developed for full aircraft configurations at the USAFA M\&SRC and USAF SEO.

\section{Acknowledgments}

The authors would like to thank the Air Force Office of Scientific Research and Dr. Keith Bergeron of the United States Air Force Academy Modeling and Simulation Research Center for their generous financial support, as well as Dr. Scott Morton of the United States Air Force Seek Eagle Office and the DoD HPC for their access to the Arctic Region Supercomputing Center. The authors would also like to thank Dr. Robert M. Hall of the NASA Langley Research Center for providing the geometry and wind tunnel data.

\section{References}

${ }^{1}$ Erickson, G.E., and Brandon, J.M., "Low-Speed Experimental Study of the Vortex Flow Effects of a Fighter Forebody Having Unconventional Cross-Section,” AIAA Paper 85-1798-CP, 1985.

${ }^{2}$ Rao, D.M., and Bhat, M.K., "A Low-Speed Wind Tunnel Study of Vortex Interaction Control Techniques on a ChineForebody/Delta-Wing Configuration,” NASA Contractor Report, NASA-CR-189616, March 1992.

${ }^{3}$ LeMay, S., and Rogers, L., "Pneumatic Vortex Flow Control on a 55-Degree Cropped Delta Wing with Chined forebody," AIAA $16^{\text {th }}$ Aerodynamic Ground Testing Conference, AIAA-90-1430, June 1990.

${ }^{4}$ Grismer, D., and Nelson, R., "The Aerodynamic Effects of Sideslip on Double Delta Wings," $31^{\text {st }}$ Aerospace Sciences Meeting and Exhibit, AIAA-93-0053, January 1993.

${ }^{5}$ Rao, D.M., and Bhat, M.K., "Subsonic Investigations of Vortex Interaction Control For Enhanced High-Alpha Aerodynamics of a Chine Forebody/ Delta Wing Configuration," NASA Contractor Report, NASA-CR-189641, June 1992.

${ }^{6}$ Rao, D.M., and Bhat, M.K., "High-Alpha Vortex Decoupling Investigations on a Chine Forebody/Delta Wing Configuration at Transonic Mach Numbers," NASA Contractor Report, NASA-CR-189642, June 1992.

${ }^{7}$ Hall, R.M., "Impact of Fuselage Cross Section on the Stability of a Generic Fighter," $16^{\text {th }}$ Applied Aerodynamics Conference, AIAA-98-2725, 1998.

${ }^{8}$ Cummings, R. M., Schiff, L. B., and Duino, J. D., "Experimental Investigation of Tangential Slot Blowing on a Generic Chined Forebody," Journal of Aircraft, Vol. 32, No. 4, July-August 1995.

${ }^{9}$ Ravi R. and Mason W.H., "A Computational Examination of Directional Stability for Smooth and Chined Forebodies at High- $\alpha, ”$ NASA Contractor Report, NASA-CR-4465, 1992.

${ }^{10}$ Ravi R. and Mason W.H., "Chine-Shaped Forebody Effects on Directional Stability at High- $\alpha$," Journal of Aircraft, Vol. 31, No. 3, May - June 1994.

${ }^{11}$ Agosta-Greenman, R. M., Gee, K., Cummings, R. M., and Schiff, L. B., "Computational Investigation of Tangential Slot Blowing Generic Chined Forebody, Journal of Aircraft, Vol. 32, No. 4, July-August 1995.

${ }^{12}$ Ghaffari, F., "On the Vortical-Flow Prediction Capability of an Unstructured-Grid Euler Solver," $32{ }^{\text {nd }}$ Aerospace Sciences Meeting and Exhibit, AIAA-94-0163, January 1994.

${ }^{13}$ Kinard, T. A., Harris, B. W., and Raj, P., "Computational Simulation of Benign and Burst Vortex Flows," $13^{\text {th }}$ AIAA Applied Aerodynamics Conference, AIAA-95-1815-CP, June 1995.

${ }^{14}$ Finley, D.B., and Karman S.L. Jr., "Application of Splitflow Unstructured CFD Code to Euler Predictions of a Generic Fighter Model," $13^{\text {th }}$ AIAA Applied Aerodynamics Conference, AIAA-95-1821-CP, June 1995.

${ }^{15}$ Finley, D.B., "Euler Technology Assessment Program for Preliminary Aircraft Design Employing SPLITFLOW Code With Cartesian Unstructured Grid Method," NASA Contractor Report, NASA-CR-4649, March 1995.

${ }^{16}$ Kinard, T.A., Harris, B.W., and Raj, P., "An Assessment of Viscous Effects in Computational Simulation of Benign and Burst Vortex Flows on Generic Fighter “wind-Tunnel Models Using TEAM Code,” NASA Contractor Report, NASA-CR-4650, March 1995.

${ }^{17}$ Treiber, D.A., and Muilenburg D.A., "Euler Technology Assessment for Preliminary Aircraft Design Employing OVERFLOW Code With Multiblock Structured-Grid Method,” NASA Contractor Report, NASA-CR-4651, March 1995.

${ }^{18}$ Kinard, T.A., and Raj, P., "Euler Technology Assessment for Preliminary Aircraft Design - Compressibility Predictions by Employing the Unstructured Grid USM3D Code," NASA Contractor Report, NASA-CR-4711, March 1996.

${ }^{19}$ Pirzadeh, S.Z., "A Solution-Adaptive Unstructured Grid Method by Grid Subdivision and Local Remeshing," Journal of Aircraft, Vol.37, No. 5, September-October 2000.

${ }^{20}$ Raj, P., Finley, D.B., and Ghaffari, F., "An Assessment of CFD Effectiveness for Vortex-Flow Simulation to Meet Preliminary Design Needs Part A - Vortex Flows and High Angle of Attack," Paper No. 47, NATO/RTO/AVT-Panel, May 2001.

${ }^{21}$ Spalart P.R., Deck S., Shur M.L., Squires K.D., Strelets, M.Kh., and Travin, A., "A New Version of Detached-Eddy Simulation, Resistant to Ambiguous Grid Densities," Theoretical Computational Fluid Dynamics, Vol. 20, 2006, pg. 181-195.

${ }^{22}$ Strang, W.Z., Tomaro, R.F., Grismer, M.J., "The Defining Methods of Cobalt60: A Parallel, Implicit, Unstructured Euler/Navier Stokes Flow Solver,” 37 $7^{\text {th }}$ AIAA Aerospace Sciences Meeting and Exhibit, AIAA Paper 99-0786, January 1999. 
${ }^{23}$ Gottlieb, J.J., and Groth, C.P.T., "Assessment of Riemann Solvers for Unsteady One-Dimensional Inviscid Flows of Perfect Gasses," Journal of Computational Physics, Vol. 78, No.2, 1988, pp. 437-458.

${ }^{24}$ Tomaro, R.F., Strang, W.Z., Sankar, L.N., "An Implicit Algorithm for Solving Time Dependent Flows On Unstructured Grids," $35^{\text {th }}$ AIAA Aerospace Sciences Meeting \& Exhibit, AIAA Paper 97-0333, January 1999.

${ }^{25}$ Forsythe, J.R., Squires, K.D., Wurtzler, K.E., and Spalart, P.R., "Detached Eddy Simulation of Fighter Aircraft at High Alpha," $40^{\text {th }}$ AIAA Aerospace Sciences Meeting and Exhibit, AIAA Paper 2002-0591, January 2002.

${ }^{26}$ Morton, S.A., Steenman, M.B., Cummings, R.M., Forsythe, J.R., "DES Grid Resolution Issues for Vortical Flows on a Delta Wing and an F18C," 41 ${ }^{\text {st }}$ AIAA Aerospace Sciences Meeting and Exhibit, AIAA Paper 2003-1103, January 2003.

${ }^{27}$ Mitchell, A.M., Morton, S.A., Forsythe, J.R., and Cummings, R.M., “Analysis of Delta-Wing Vortical Substructures Using Detached-Eddy Simulation," AIAA Journal, Vol. 44, No.5, May 2006.

${ }^{28}$ Spalart, P.R., Jou, W.H., Strelets, M. and Allmaras, S.R., "Comments on the Feasibility of LES for Wings, and on a Hybrid RANS/LES approach," Advances in DNS/LES, $1^{\text {st }}$ AFOSR International Conference on DNS/LES, Greyden Press, Columbus. $\mathrm{OH}, 1997$.

${ }^{29}$ Spalart, P.R., and Allmaras, S.R., "A One Equation Turbulence Model for Aerodynamic Flows," La Recherche Aerospatiale, Vol. 1, 1994, pp. 5-21.

${ }^{30}$ Menter, F.R., Kuntz, M., "Adaptation of Eddy-Viscosity Turbulence Models to Unsteady separated Flow Behind Vehicles," In: McCallen, R., Browand, F., Ross, J. (eds.) Symposium on "the aerodynamics of heavy vehicles: trucks, busses and trains." Monterey, USA, 2-6 Dec 2002, Springer, Berlin Heidelberg NewYork, 2004.

${ }^{31}$ Shur, M.L., Strelets, M.K., Travin, A.K., Spalart, P.R., "Turbulence Modeling in Rotating and Curved Channels: Assessing the Spalart-Shur Correction," AIAA Journal, Vol. 38, No. 5, May 2000.

${ }^{32}$ Mississippi State University Computational Simulation and Design Center, "Solid Mesh 5.45 3D Users Manual," July 2008.

${ }^{33}$ Cummings, R.M., Morton, S.A., McDaniel D.R., "Experiences in Accurately Predicting Time-Dependant Flows," Progress in Aerospace Sciences, Vol. 44, 2008, pp. 241-257.

${ }^{34}$ Dubief Y., and Delcayre, F., "On Coherent-Vortex Identification in Turbulence," Journal of Turbulence, Vol. 1, pg. 1-22, 2000.

${ }^{35}$ Grismer, D. S., and Nelson, R. C., "Double-Delta-Wing Aerodynamics for Pitching Motions With and Without Sideslip," Journal of Aircraft, Vol. 32, No. 6, Nov-Dec 1995. 


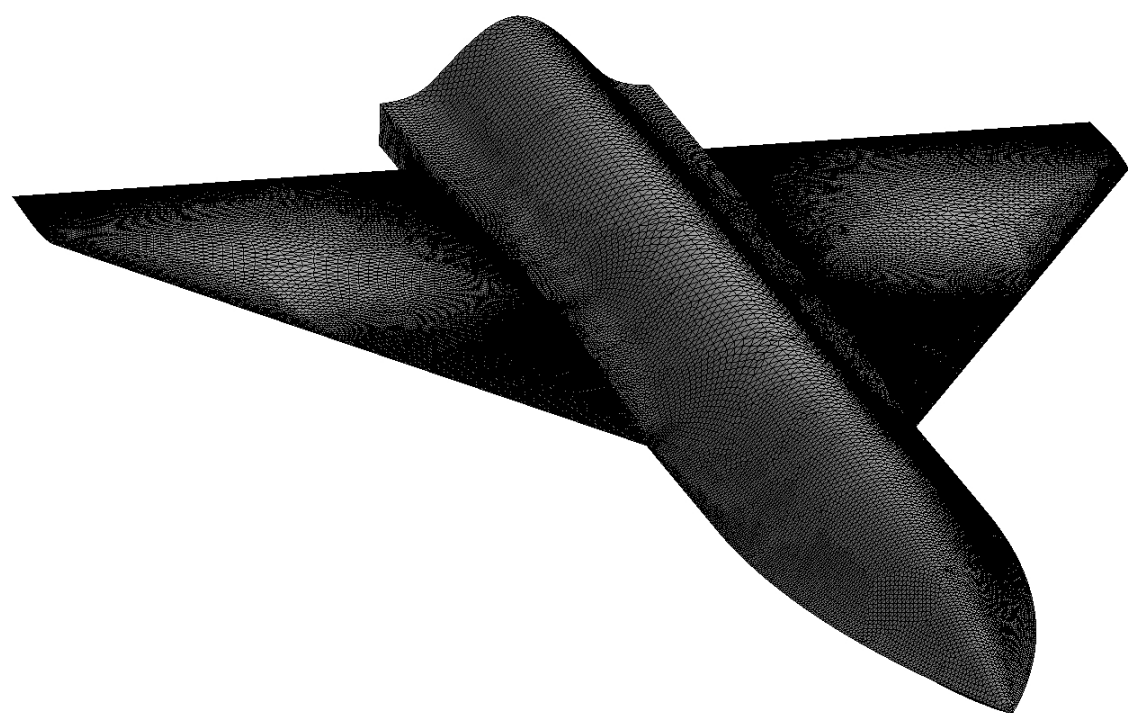

(a) Figure 1. (a) Schematic of overall MTVI configuration and surface mesh. (b) Schematic of forebody cross section with $30^{\circ}$ chine.

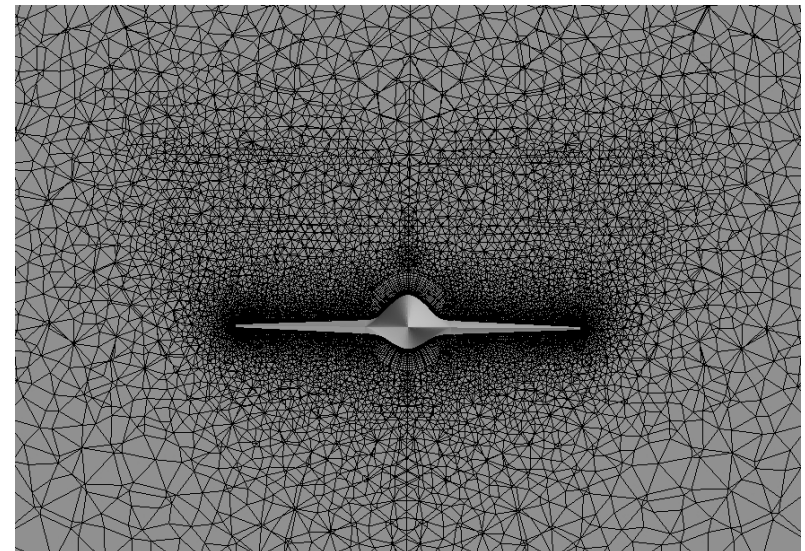

(a)

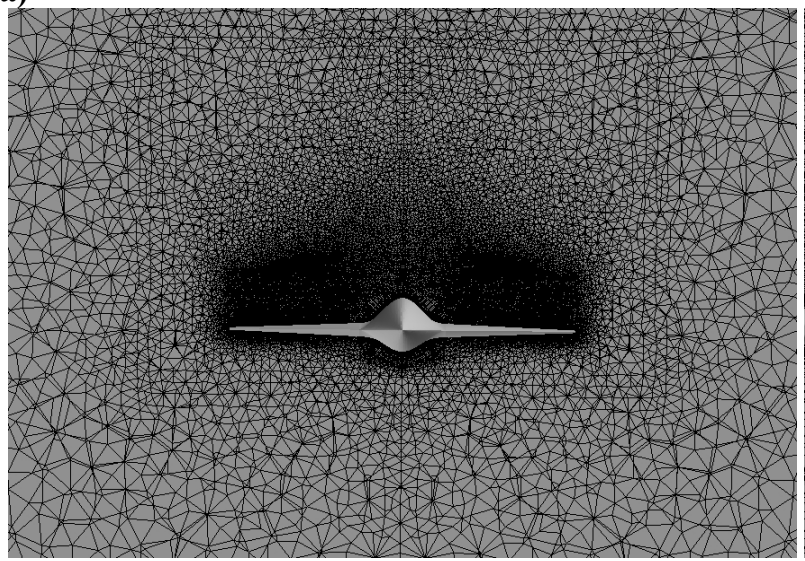

(c)

Figure 2. (a) Cross sectional view ( $x-y$ plane) of the medium density mesh at $x / l=0.88$. (b) Side view of the medium density mesh near the $y-z$ plane $(y / l=0.06)$. (c) Cross sectional view $(x-y$ plane) of the very fine mesh at $x / l=0.88$. (d) Side view of the very fine mesh near the $y-z$ plane $(y / l=0.06)$.

American Institute of Aeronautics and Astronautics 092407

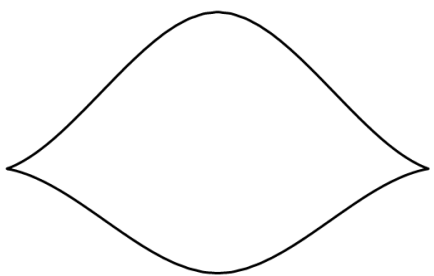

(b)

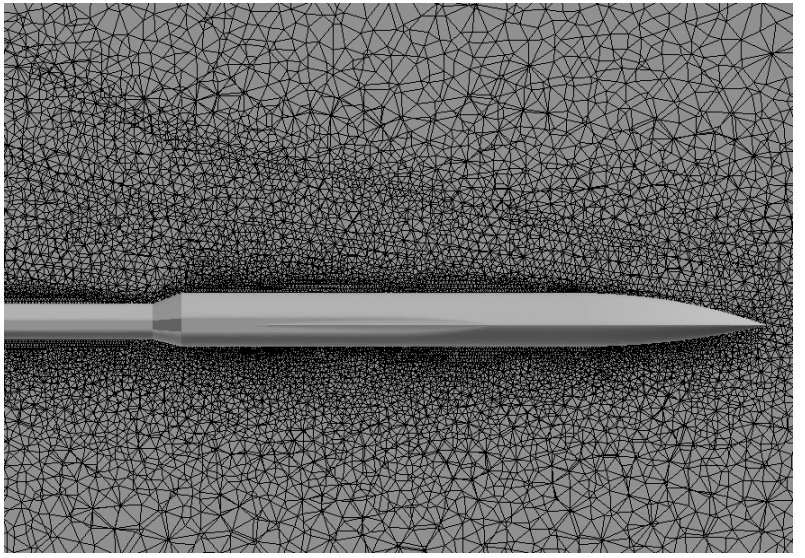

(b)

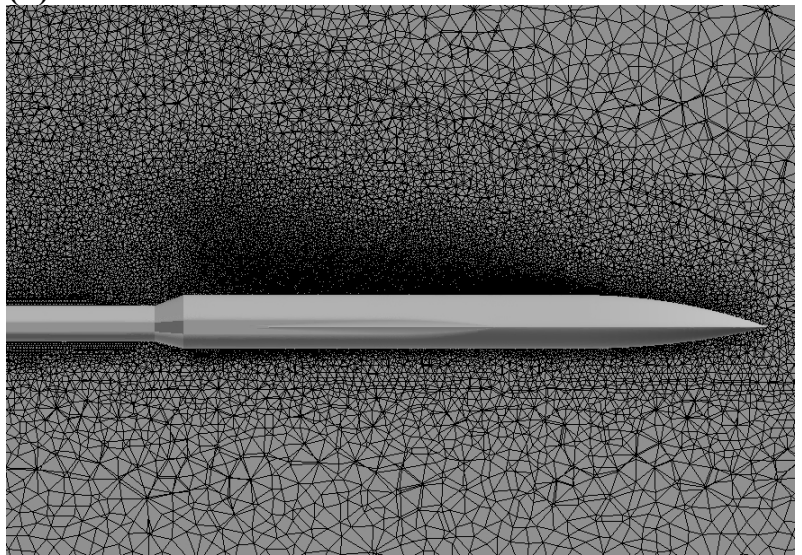

(d) 


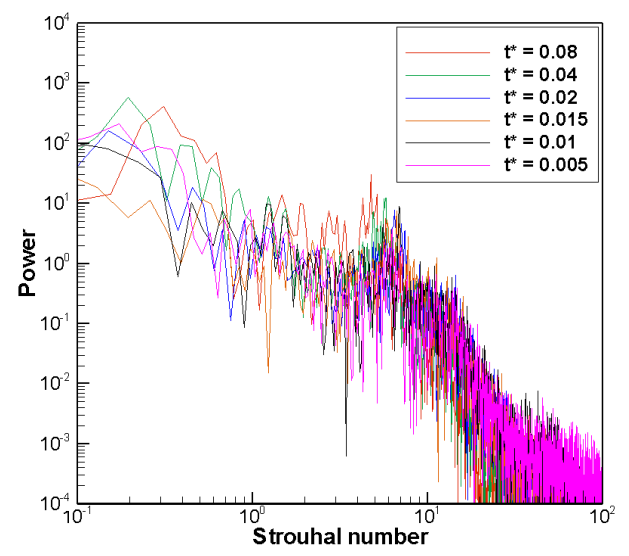

(a)

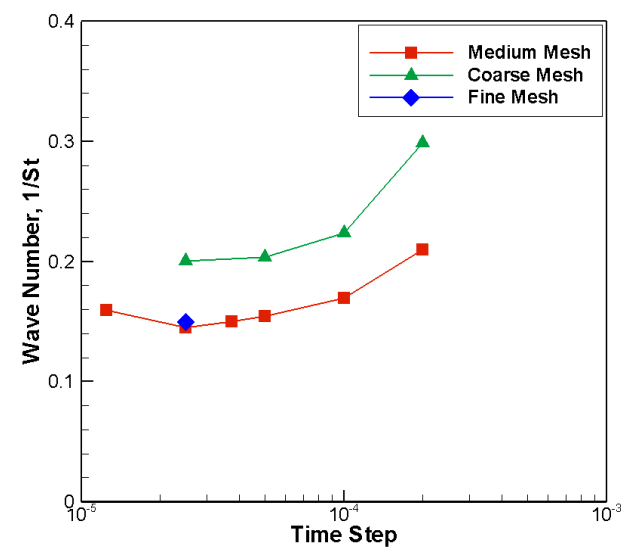

(b)

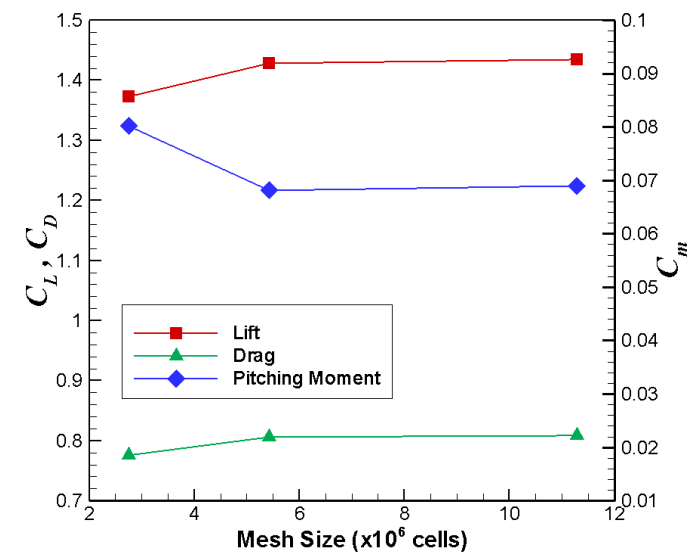

(c)

Figure 3. (a) Variation of the pitching moment power spectrum density with time step for the medium density mesh. (b) Pitching moment wave number variation with time step for the low, medium and high density meshes. (c) Variation of time averaged force and moment predictions with mesh size for $t^{*}=0.01$.

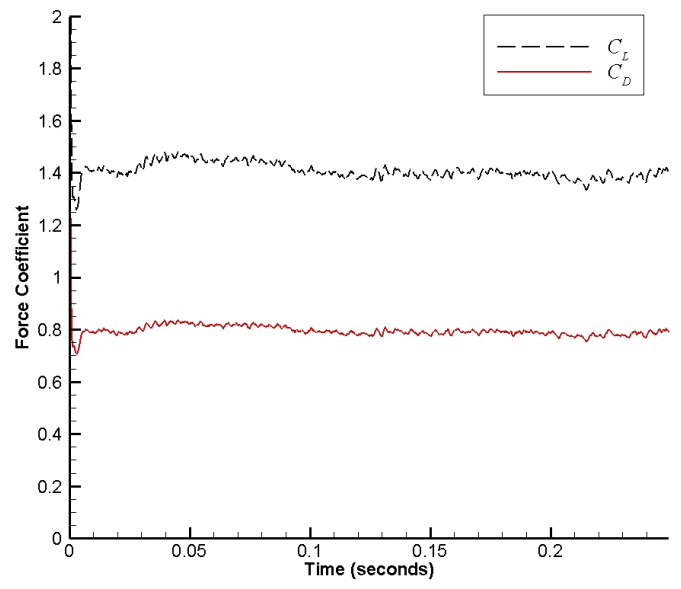

(a)

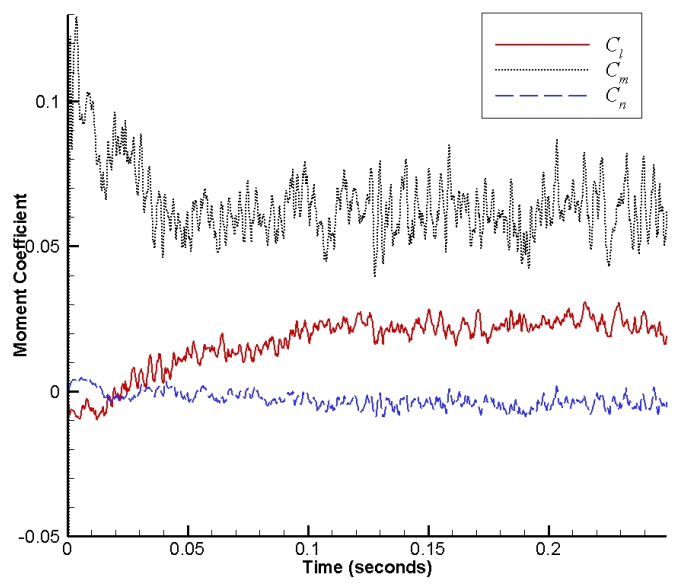

(b)

Figure 4. A time history of CFD predicted (a) force coefficients and (b) moment coefficients for $\alpha=30^{\circ}$ and $\beta$ $=\mathbf{1 . 0 0}{ }^{\circ}$.

American Institute of Aeronautics and Astronautics 


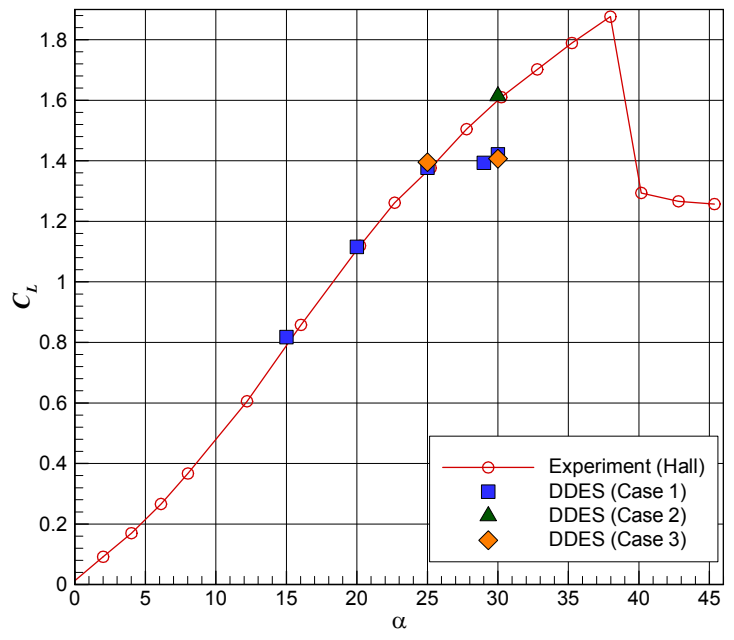

(a) Lift.

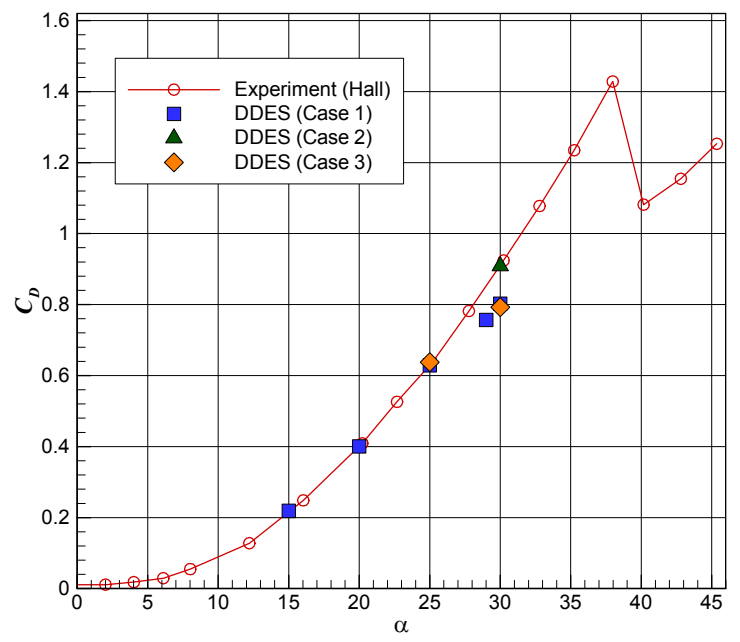

(b) Drag.

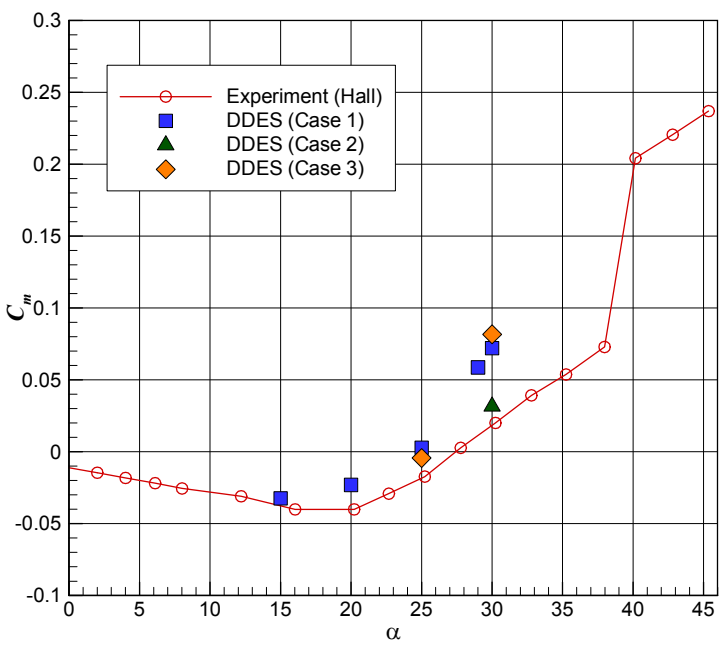

(c) Pitching moment.

Figure 5. A comparison of CFD predicted and experimental lift, drag, and pitching moment coefficient as a function of $\alpha$ for zero sideslip at $M_{\infty}=0.4$, and $\operatorname{Re}_{\bar{c}}=2.68 \times 10^{6}$.

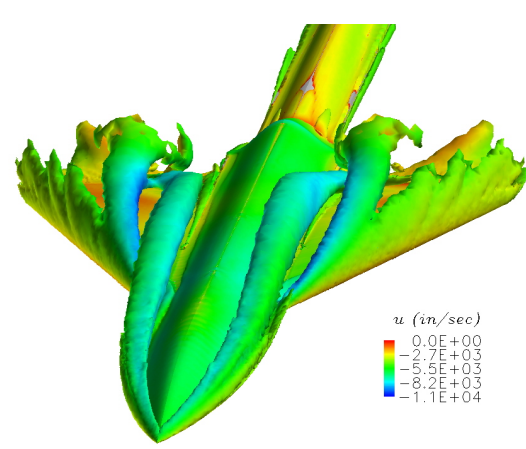

(a) Case 1

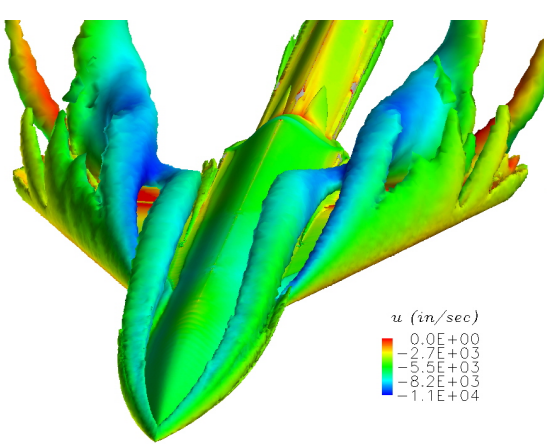

(b) Case 2

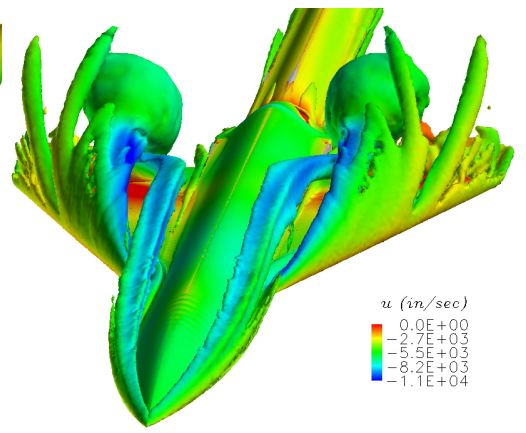

(c) Case 3

Figure 6. Isosurfaces of $Q=1.0 \times 10^{7} \mathrm{~s}^{-2}$ for the various DDES simulations at $\alpha=30.0^{\circ}$ and $\beta=0.0^{\circ}$. The isosurface is colored by axial velocity with red corresponding to $u=0$ and blue corresponding to $u=-2 u_{\infty}$.

American Institute of Aeronautics and Astronautics 092407 


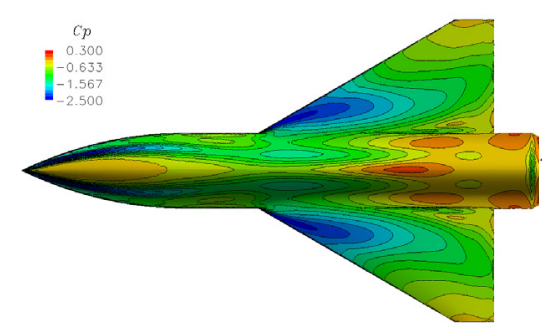

(a) Case 1

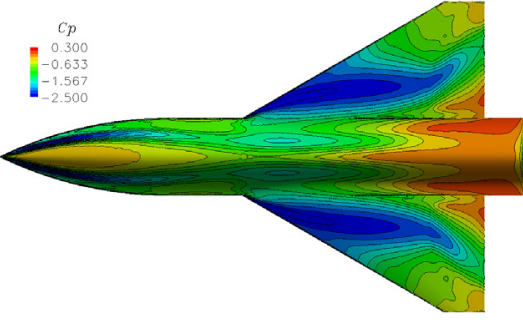

(b) Case 2

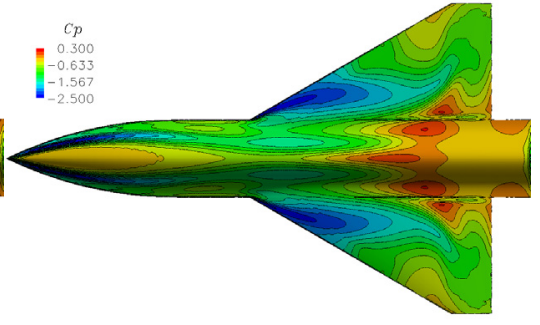

(c) Case 3

Figure 7. A top view of surface pressure contours for the various DDES simulations at $\alpha=30.0^{\circ}$ and $\beta=0.0^{\circ}$. Red corresponds to $\mathrm{C} p=\mathbf{0 . 3}$ and blue corresponds to $\mathrm{Cp}=\mathbf{- 2 . 5}$.

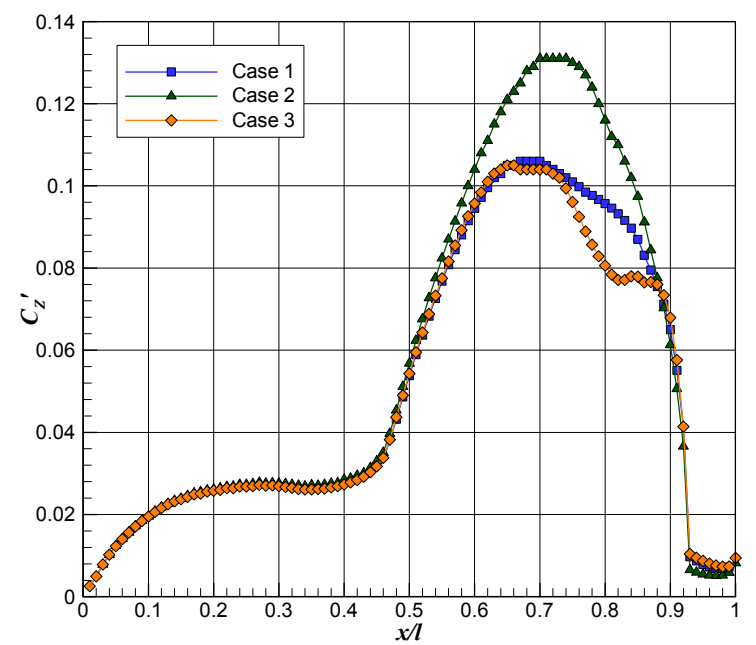

Figure 8. Axial distributions of the body force in the z-axis direction for the various simulations at $\alpha=30.0^{\circ}$ and $\beta=0.0^{\circ}$.

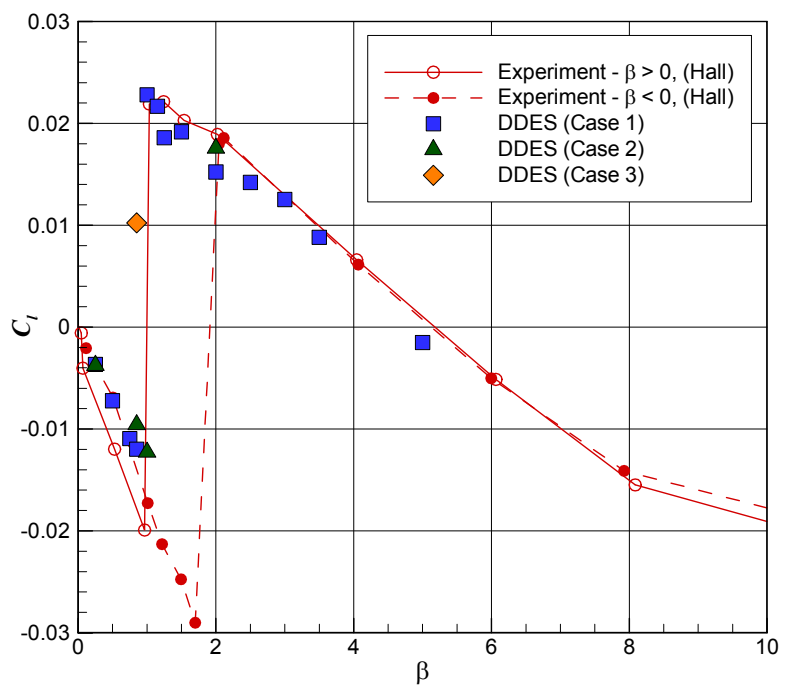

(a)

Figure 9. A comparison of CFD predicted and experimental rolling and yawing moment coefficient as a function of $\beta$ for $\alpha=30^{\circ}, M_{\infty}=0.4$, and $\operatorname{Re}_{\bar{c}}=2.68 \times 10^{6}$.

American Institute of Aeronautics and Astronautics 092407

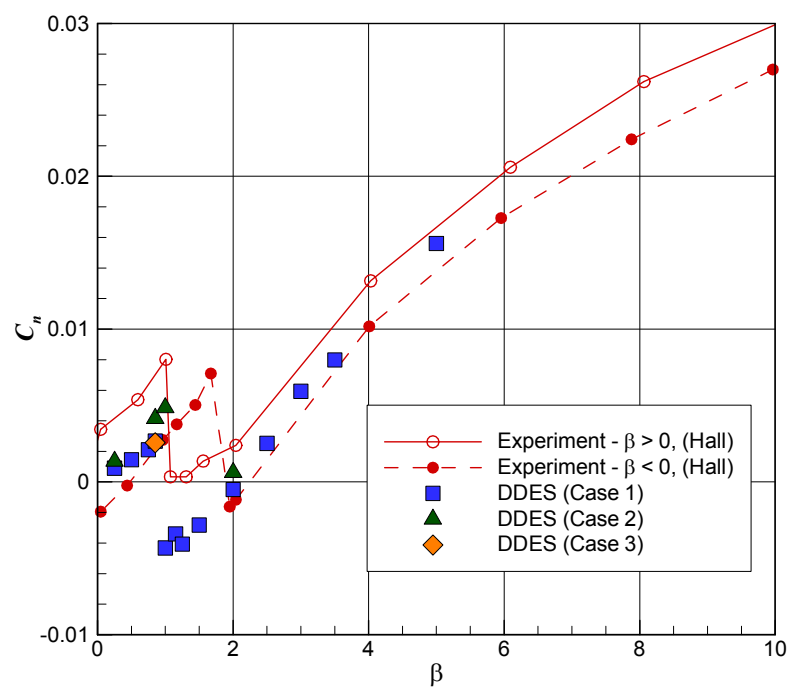

(b) 


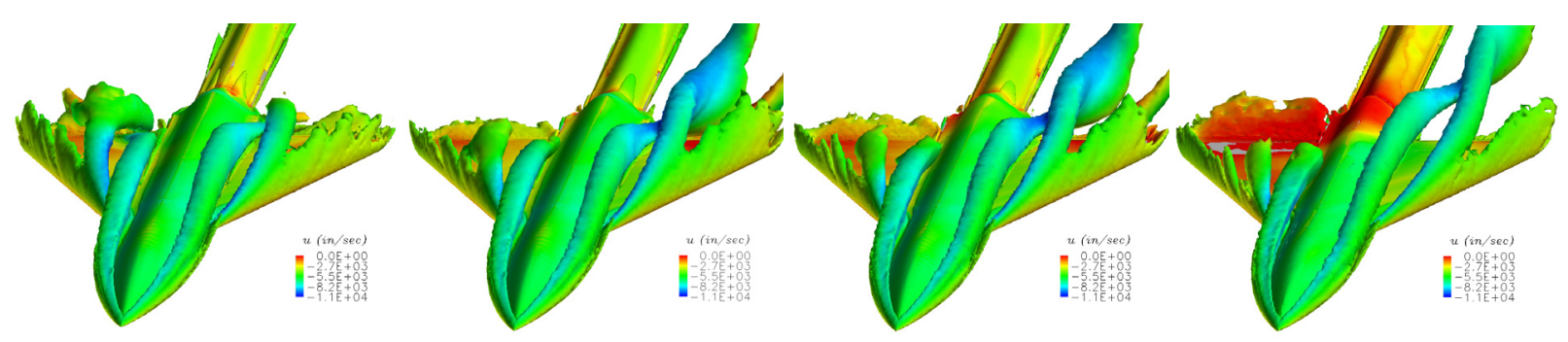
(a) $\beta=0.85^{\circ}$
(b) $\beta=1.0^{\circ}$
(c) $\beta=2.00^{\circ}$
(d) $\boldsymbol{\beta}=\mathbf{5 . 0 0 ^ { \circ }}$

Figure 10. Isosurfaces of $Q=1.0 \times 10^{7} \mathrm{~s}^{-2}$ for Case 1 simulations at various $\beta$. The isosurface is colored by axial velocity with red corresponding to $u=0$ and blue corresponding to $u=-2 u_{\infty}$.
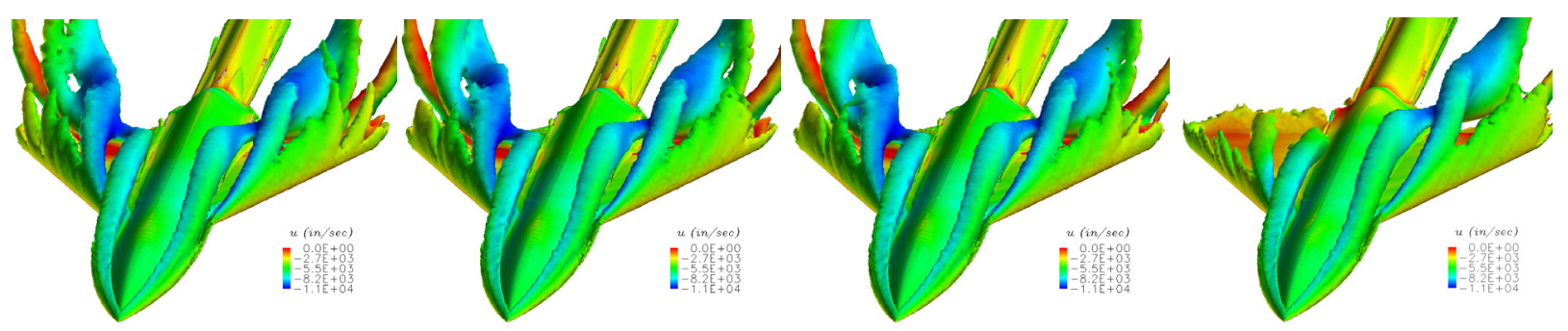

(a) $\beta=0.25^{\circ}$

(b) $\beta=0.85^{\circ}$

(c) $\beta=1.00^{\circ}$

(d) $\beta=\mathbf{2 . 0 0 ^ { \circ }}$

Figure 11. Isosurfaces of $Q=1.0 \times 10^{7} \mathrm{~s}^{-2}$ for Case 2 simulations at various $\beta$. The isosurface is colored by axial velocity with red corresponding to $u=0$ and blue corresponding to $u=-2 u_{\infty}$.
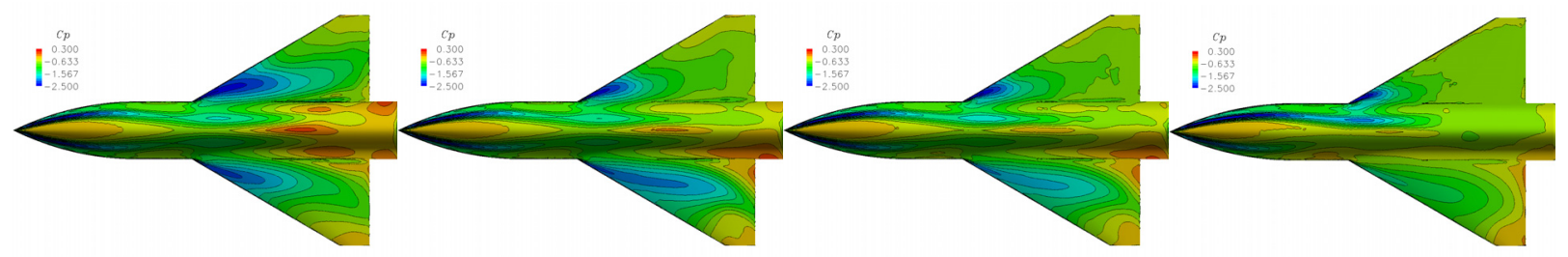

(a) $\beta=0.85^{\circ}$

(b) $\beta=1.0^{\circ}$

(c) $\beta=2.00^{\circ}$

(d) $\beta=5.00^{\circ}$

Figure 12. A top view of surface pressure contours for Case 1 simulations at various $\beta$. Red corresponds to $\mathrm{C} p=0.3$ and blue corresponds to $\mathrm{C} p=-2.5$.

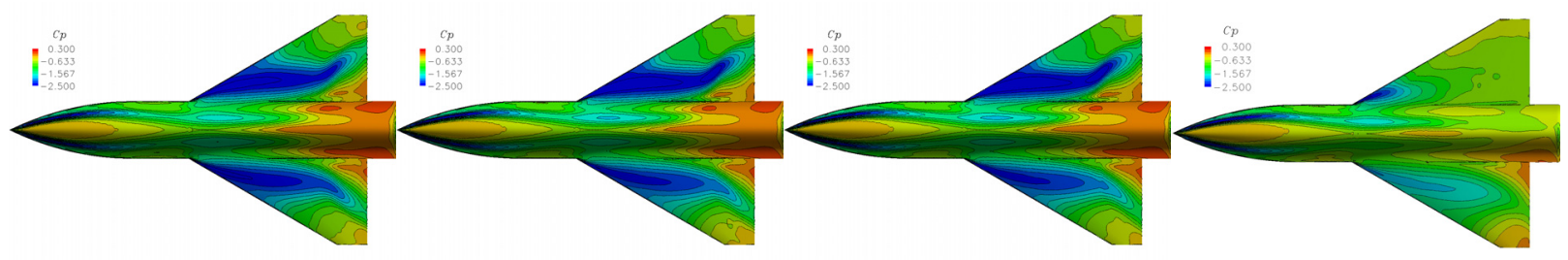
a) $\boldsymbol{\beta}=\mathbf{0 . 2 5 ^ { \circ }}$
(b) $\beta=0.85^{\circ}$
(c) $\beta=1.00^{\circ}$
(d) $\beta=\mathbf{2 . 0 0}{ }^{\circ}$

Figure 13. A top view of surface pressure contours for Case 2 simulations at various $\beta$. Red corresponds to $\mathrm{C} p=0.3$ and blue corresponds to $\mathrm{Cp}=\mathbf{- 2 . 5}$. 


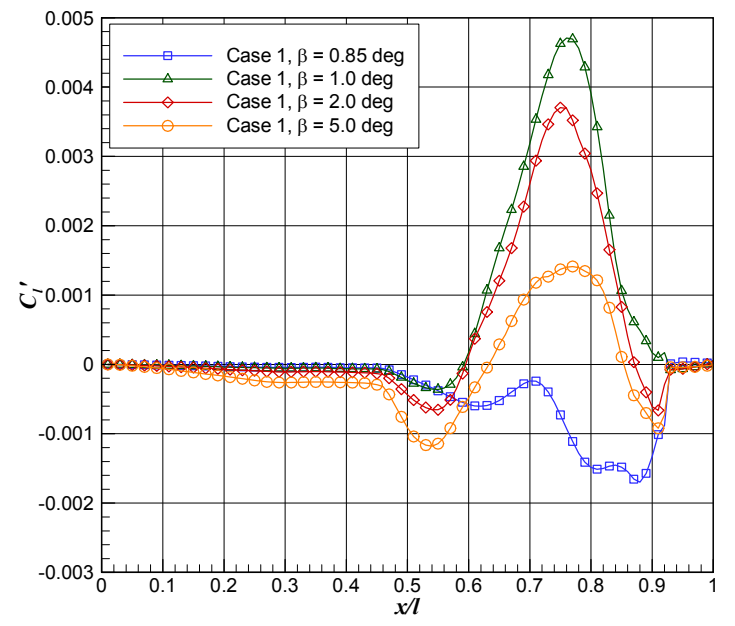

(a) Case 1

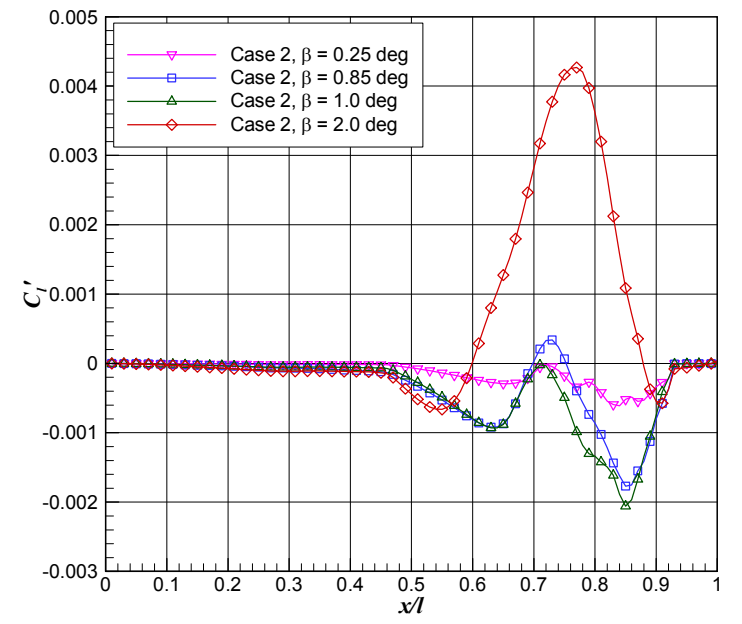

(b) Case 2

Figure 14. Axial distributions of rolling moment, $C_{l}$, at various $\beta$.

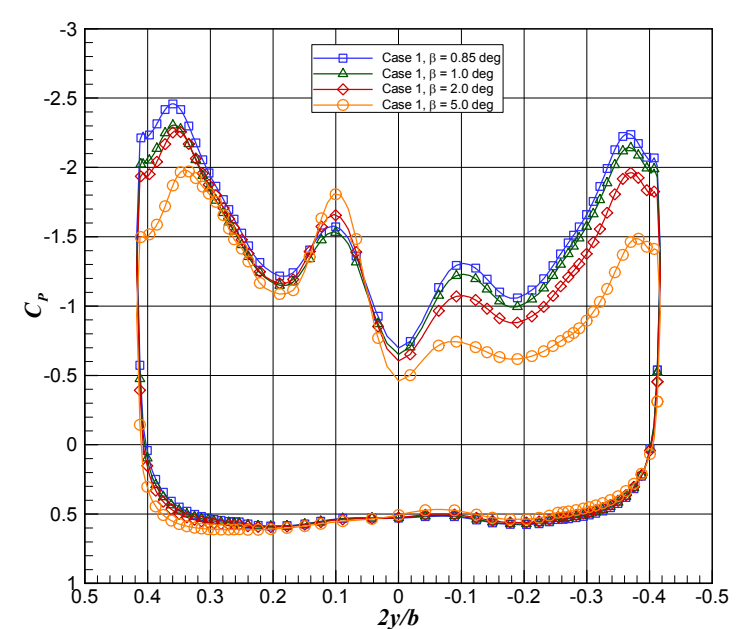

(a) $x / l=0.55$

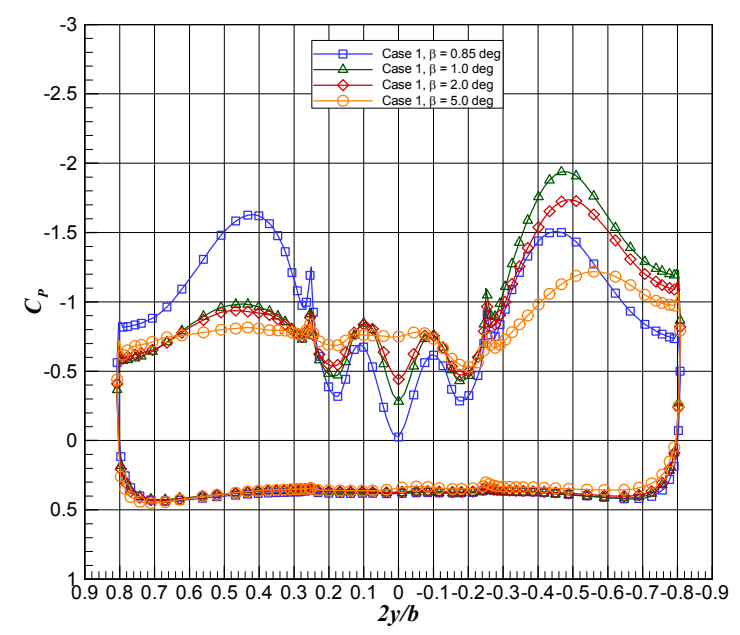

(c) $x / l=0.75$

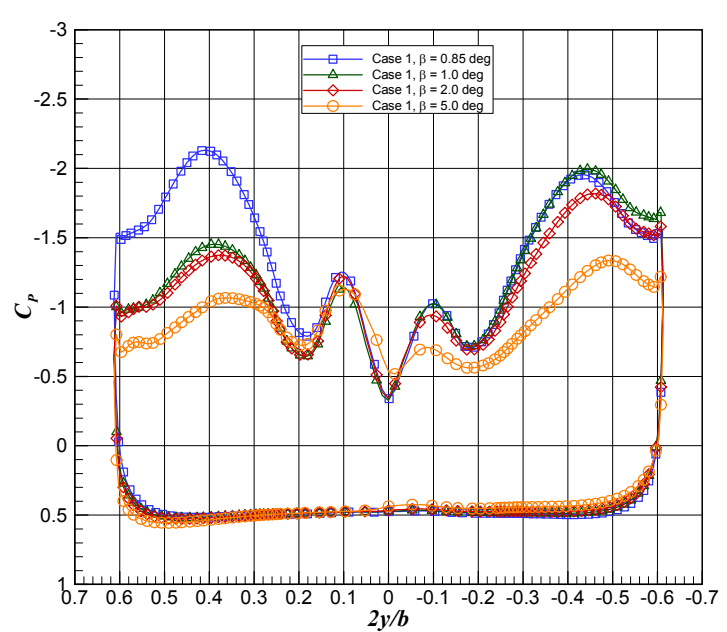

(b) $x / l=0.65$

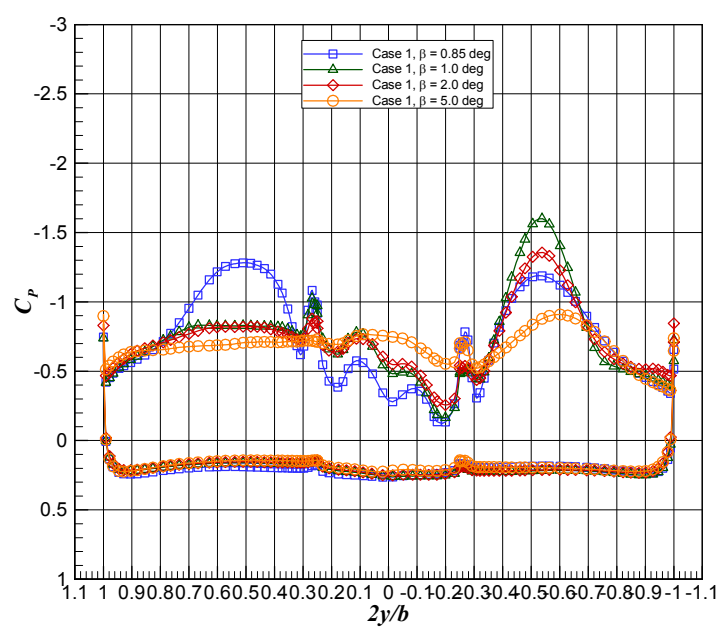

(d) $x / l=0.85$

Figure 15. Pressure distributions at constant axial locations for Case 1 simulations at various $\beta$. Every $5^{\text {th }}$ datum point shown.

American Institute of Aeronautics and Astronautics DISTRIBUTION A. Approved for public release, distribution unlimited. 092407 


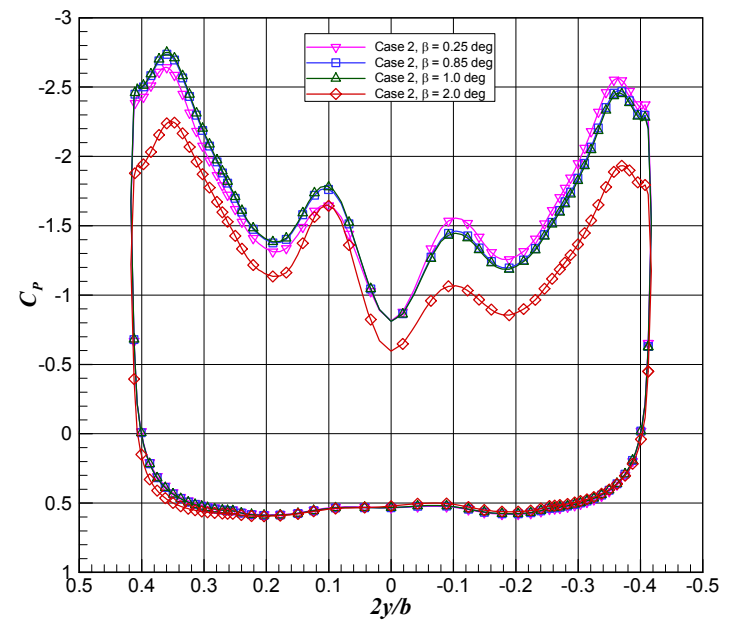

(a) $x / l=0.55$

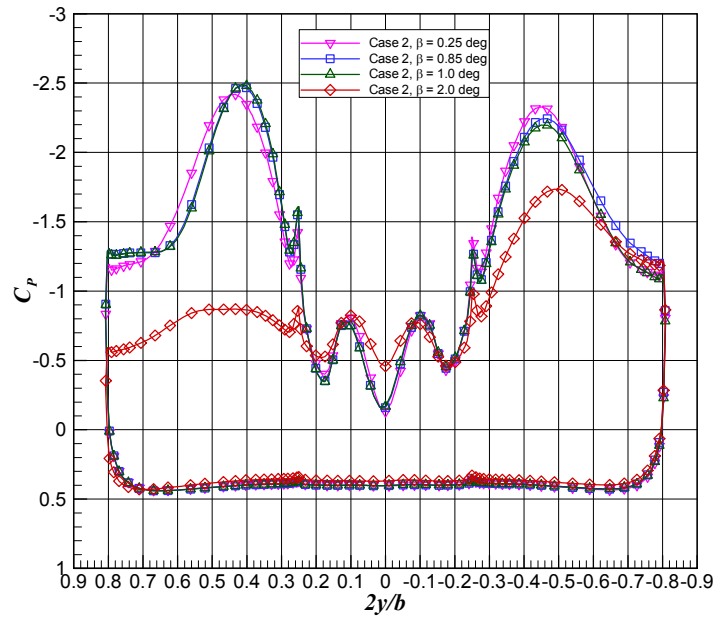

(c) $x / l=0.75$

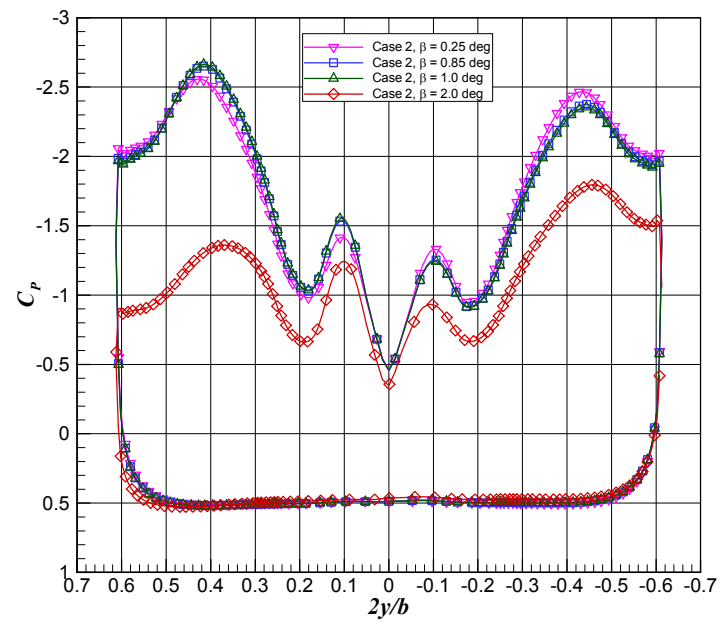

(b) $x / l=0.65$

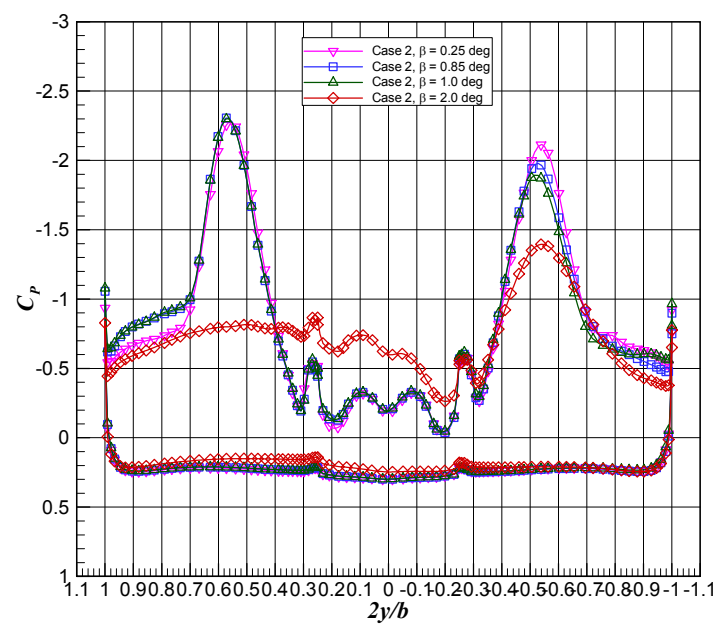

(d) $x / l=0.85$

Figure 16. Pressure distributions at constant axial locations for Case 2 simulations at various $\beta$. Every $5^{\text {th }}$ datum point shown.

American Institute of Aeronautics and Astronautics 

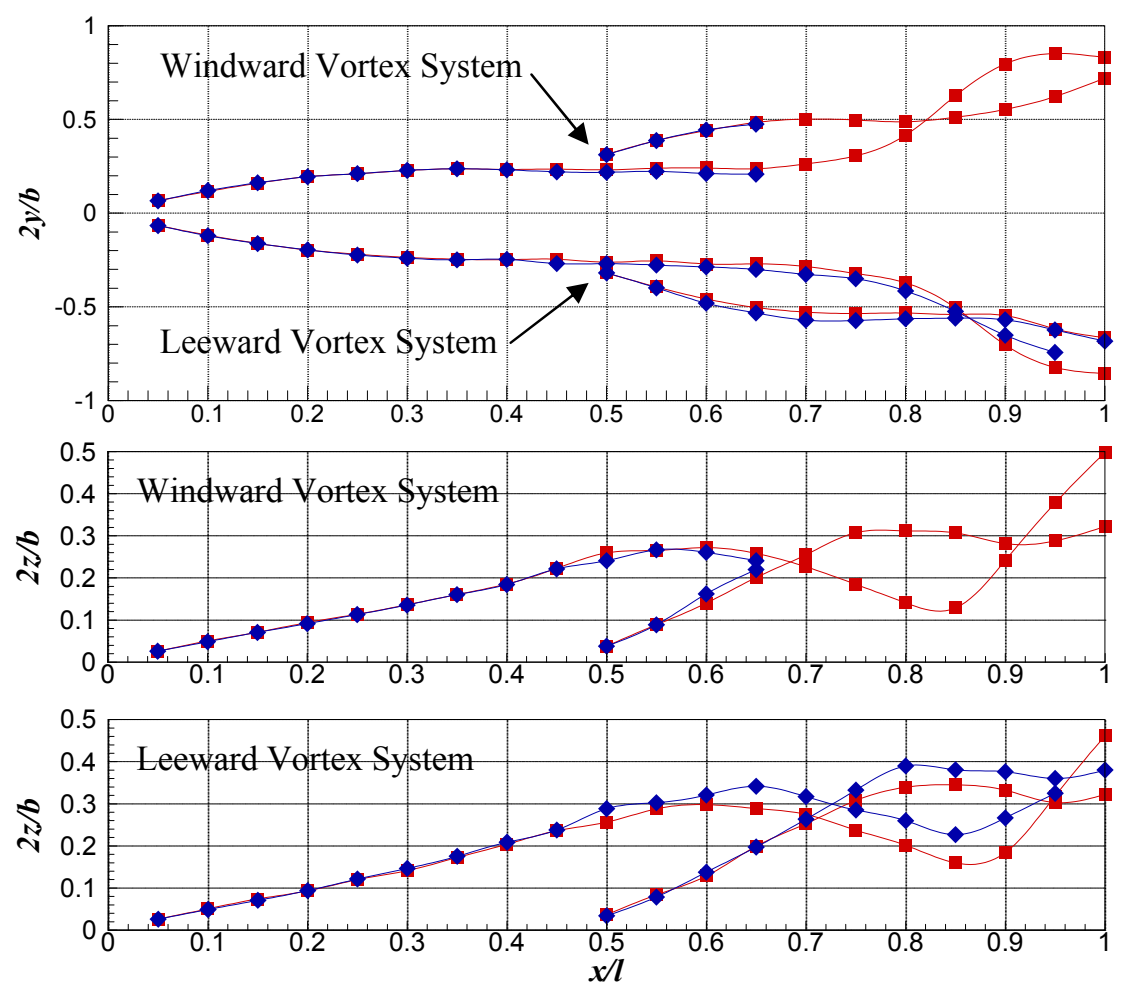

Figure 17. Vortex Trajectories based on minimum total pressure for Case 2 simulations at $\beta=1.00^{\circ}$ and $2.00^{\circ}$. Red squares represent $\beta=\mathbf{1 . 0 0}^{\circ}$ and blue diamonds represent $\beta=\mathbf{2 . 0 0}^{\circ}$.
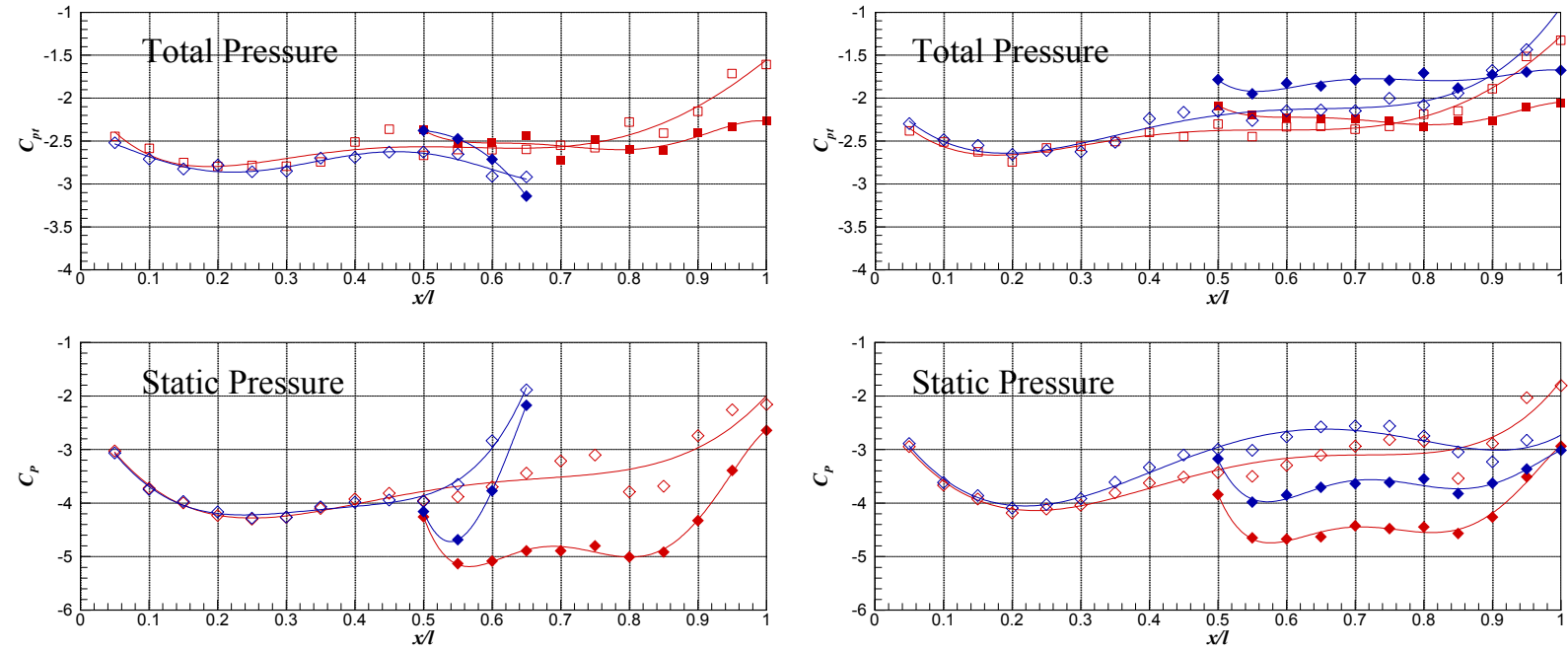

(a) Windward vortex system.

(b) Leeward vortex system.

Figure 18. DDES predictions of total pressure and static pressure coefficient through the vortex cores of Case 2 simulations at $\beta=1.00^{\circ}$ and $2.00^{\circ}$. Red squares represent $\beta=1.00^{\circ}$, blue diamonds represent $\beta=2.00^{\circ}$, unfilled symbols represent the chine vortex, and filled symbols represent the wing vortex. 